\title{
The Choquard Equation with Weighted Terms and Sobolev-Hardy Exponent
}

\author{
Yanbin Sang $\mathbb{D}^{1,},{ }^{1,2}$ Xiaorong Luo, ${ }^{1}$ and Yongqing Wang $\mathbb{D}^{2}$ \\ ${ }^{1}$ Department of Mathematics, School of Science, North University of China, Taiyuan, Shanxi 030051, China \\ ${ }^{2}$ School of Mathematical Sciences, Qufu Normal University, Qufu, Shandong 273165, China \\ Correspondence should be addressed to Yanbin Sang; syb6662004@163.com
}

Received 24 March 2018; Accepted 27 May 2018; Published 8 July 2018

Academic Editor: Gennaro Infante

Copyright (c) 2018 Yanbin Sang et al. This is an open access article distributed under the Creative Commons Attribution License, which permits unrestricted use, distribution, and reproduction in any medium, provided the original work is properly cited.

We study a nonlinear Choquard equation with weighted terms and critical Sobolev-Hardy exponent. We apply variational methods and Lusternik-Schnirelmann category to prove the multiple positive solutions for this problem.

\section{Introduction}

The main goal of this paper is to consider the multiplicity of positive solution for the following nonlinear Choquard equation:

$$
\begin{aligned}
&-\Delta u= g(x)\left(\int_{\Omega} \frac{|u|^{2_{\mu}^{*}}}{|x-y|^{\mu}} d y\right)|u|^{2_{\mu}^{*}-2} u \\
&+f_{\lambda}(x)|u|^{q-2} u, \text { in } \Omega, \\
& u=0, \quad \text { on } \partial \Omega,
\end{aligned}
$$

where $\Omega \subset \mathbb{R}^{N}$ is a bounded open set with smooth boundary. $N>3,0<\mu<N, 1<q<2,2_{\mu}^{*}=(2 N-\mu) /(N-2)$, and $\lambda>0$ is a parameter. There are two continuous weight functions, $f_{\lambda}, g$ satisfying the following conditions:

$$
\begin{aligned}
& \left(f_{1}\right) f_{\lambda}=\lambda f_{+}+f_{-},\left(f_{ \pm}= \pm \max \{ \pm f, 0\} \neq \equiv 0\right) . \\
& \left(g_{1}\right) g \in C(\bar{\Omega}) \text { and } g_{+}=\max \{g, 0\} \neq \equiv 0 .
\end{aligned}
$$

$\left(f_{2}\right)$ there exist two positive constants $\beta_{0}, \rho_{0}$ such that $B\left(0,2 \rho_{0}\right) \subset \Omega$ and $f_{\lambda}(x) \geq \beta_{0}$ for every $x \in B\left(0,2 \rho_{0}\right)$. $\left(g_{2}\right)\left|g_{+}\right|_{\infty}=g(0)=\max _{x \in \bar{\Omega}} g(x), \forall x \in$ $B\left(0,2 \rho_{0}\right), g(x)>0$, there exists $\beta(\beta>N-\mu)$ such that

$$
g(x)-g(0)=o\left(|x|^{\beta}\right), \quad(x \longrightarrow 0) .
$$

In recent years, much attention has been paid to nonlinear Choquard equation involving the Hardy-Littlewood-Sobolev inequality, which generalizes and complements the classical elliptic boundary value problems and Schrödinger-Poisson system [1-21]. In particularly, more and more authors have studied the critical problems [22-36]. In the case that $g(x)=$ $f_{\lambda}(x)=1$, Gao and Yang [22] considered the existence and multiplicity of (1) with upper critical exponent $2_{\mu}^{*}=$ $(2 N-\mu) /(N-2)$. The authors focus on the case that how the sublinear perturbation term has influence on the multiplicity of (1). When $2_{\mu}^{*}>2, g(x)=1, f_{\lambda}(x)=0$, Xiang [33] showed the uniqueness and nondegeneracy solutions for ground states of Choquard equation. Furthermore, by means of the tool of Nehari manifold, Zhang et al. [36] established the existence theorem of ground states for generalized Choquard equation when the nonlinear term is concave-convex. On the other hand, there are a great deal of results on the existence of elliptic boundary value problems with sign-changing weights [37-45]. We should point out that Hsu and Lin [38] showed the existence of positive solutions for elliptic equations with concave-convex nonlinearities and sign-changing weights. What is more, $\mathrm{Wu}$ [39] obtained three positive solutions discussed in [38] by using of the method of Nehari manifold combining with the Lusternik-Schnirelmann category. In view of the same method, Chen and $\mathrm{Wu}$ [41] obtained the existence of positive solutions for a class of critical semilinear problem. Chen et al. [42] established multiplicity 
theorems for Kirchhoff type equation with sign-changing weight functions. Goyal and Sreenadh [45] used fibering map approach to study $p$-fractional Laplacian equation with signchanging weight function.

Motivated by above results, we use variational approach to analyze the existence and multiplicity of positive solutions for (1). To the best of our knowledge, there is no result studying Choquard equation with upper critical exponent and two sign-changing weight functions.

Set

$$
\begin{aligned}
\lambda_{*}= & \left(\frac{2-q}{22_{\mu}^{*}-q}\left|g_{+}\right|_{\infty}^{-1}\right)^{(2-q) /\left(22_{\mu}^{*}-q\right)}\left(\frac{22_{\mu}^{*}-2}{22_{\mu}^{*}-q}\left|f_{+}\right|_{\infty}^{-1}\right) \\
& \cdot|\Omega|^{\left(q-2^{*}\right) / 2^{*}} C(N, \mu)^{q / 22_{\mu}^{*}} S_{H, L}^{\left(2_{\mu}^{*}(2-q) / 22_{\mu}^{*}\right)+(q / 2)} .
\end{aligned}
$$

Our main results are the following theorems.

Theorem 1. Assume that $\left(f_{1}\right),\left(g_{1}\right)$ hold, for every $\lambda \in\left(0, \lambda_{*}\right)$, the problem (1) has at least one positive solution in $H_{0}^{1}(\Omega)$.

Theorem 2. Assume that $\left(f_{1}\right),\left(g_{1}\right),\left(f_{2}\right),\left(g_{2}\right)$ hold; there exists $\lambda^{*}>0$, such that, for every $\lambda \in\left(0, \lambda^{*}\right)$, the problem (1) has at least two positive solutions in $H_{0}^{1}(\Omega)$.

Furthermore, we will utilize the following condition.

(G) There are a nonempty closed set $G=\{x \in \bar{\Omega} \mid g(x)=$ $\left.\max _{y \in \bar{\Omega}} g(y) \equiv 1\right\} \subset \Omega$ and $\beta>N-\mu$ such that

$$
g(z)-g(x)=O\left(|x-z|^{\beta}\right)
$$

holds uniformly for $z \in G$ in the limit $x \longrightarrow z$.

Remark 3. Let $G_{r}=\left\{x \in \mathbb{R}^{N} \mid \operatorname{dist}(x, G)<r\right\}$ for $r>0$. According to $(G)$, we can assume that there exist two positive solutions $D$ and $r_{1}$ such that

$$
\begin{gathered}
g(x)>0, \quad \forall x \in G_{r_{1}} \subset \Omega, \\
g(z)-g(x) \leq D|x-z|^{\beta} \quad \forall x \in B^{N}\left(z, r_{1}\right), \quad \forall z \in G .
\end{gathered}
$$

Theorem 4. Assume that $(G)$ holds; for every $\delta \in\left(0, r_{1}\right)$, there exists $\lambda_{\delta}>0$ such that for $\lambda \in\left(0, \lambda_{\delta}\right)$, (1) has at least cat $_{\mathscr{M}_{\delta}}(G)+1$ positive solutions.

Definition 5. $u \in H_{0}^{1}(\Omega)$ is called a positive weak solution of (1), if

$$
\begin{aligned}
& \int_{\Omega} \nabla u \nabla \varphi \\
&=\int_{\Omega} \int_{\Omega} g(x) \frac{|u(x)|^{2_{\mu}^{*}}|u(y)|^{2_{\mu}^{*}-2} u(y) \varphi(x)}{|x-y|^{\mu}} d x d y \\
& \quad+\int_{\Omega} f_{\lambda}(x)|u|^{q-1} \varphi, \quad \text { for every } \varphi \in H_{0}^{1}(\Omega) .
\end{aligned}
$$

The energy functional associated with the problem (1) is given by

$$
\begin{aligned}
I_{\lambda, \mu}(u)= & \frac{1}{2}\|u\|^{2} \\
& -\frac{1}{22_{\mu}^{*}} \int_{\Omega} \int_{\Omega} g(x) \frac{|u(x)|^{2_{\mu}^{*}}|u(y)|^{2_{\mu}^{*}}}{|x-y|^{\mu}} d x d y \\
& -\int_{\Omega} f_{\lambda}(x)|u|^{q},
\end{aligned}
$$

where $\|u\|^{2}=\int_{\Omega}|\nabla u|^{2} d x$ is the norm in $H_{0}^{1}(\Omega)$.

\section{Preliminaries}

Proposition 6 (Hardy-Littlewood-Sobolev inequality [22]). Let $t, r>1$ and $0<\mu<N$ with $1 / t+\mu / N+1 / r=2, f \epsilon$ $L^{t}\left(\mathbb{R}^{N}\right)$ and $h \in L^{r}\left(\mathbb{R}^{N}\right)$. There exists a constant $C(t, N, \mu, r)$ independent of $f, h$, such that

$$
\int_{\mathbb{R}^{N}} \int_{\mathbb{R}^{N}} \frac{f(x) h(y)}{|x-y|^{\mu}} d x d y \leq C(t, N, \mu, r)|f|_{t}|h|_{r} .
$$

If $t=r=2 N /(2 N-\mu)$, then

$$
C(t, N, \mu, r)=C(N, \mu)
$$

$$
=\pi^{\mu / 2} \frac{\Gamma(N / 2-\mu / 2)}{\Gamma(N-\mu / 2)}\left\{\frac{\Gamma(N / 2)}{\Gamma(N)}\right\}^{-1+\mu / N} .
$$

For $u \in D^{1,2}\left(\mathbb{R}^{N}\right)$, by Proposition 6, we have

$$
\begin{aligned}
& \left(\int_{\mathbb{R}^{N}} \int_{\mathbb{R}^{N}} \frac{|u(x)|^{2_{\mu}^{*}}|u(y)|^{2_{\mu}^{*}}}{|x-y|^{\mu}} d x d y\right)^{1 / 2_{\mu}^{*}} \\
& \quad \leq C(N, \mu)^{1 / 2_{\mu}^{*}}|u|_{2^{*}}^{2} .
\end{aligned}
$$

The best constant $S_{H, L}$ is defined as

$$
\begin{aligned}
& S_{H, L} \\
& :=\inf _{u \in D^{1,2}\left(\mathbb{R}^{N}\right)\{\{0\}} \frac{\int_{\mathbb{R}^{N}}|\nabla u|^{2} d x}{\left(\int_{\mathbb{R}^{N}} \int_{\mathbb{R}^{N}}|u(x)|^{2_{\mu}^{*}}|u(y)|^{2_{\mu}^{*}} /|x-y|^{\mu} d x d y\right)^{1 / 2_{\mu}^{*}}} .
\end{aligned}
$$

Proposition 7 (see [22]). The constant $S_{H, L}$ is attained if and only if

$$
u=C\left(\frac{a}{a^{2}+|x-b|^{2}}\right)^{(N-2) / 2},
$$

where $C>0, b \in \mathbb{R}^{N}, a \in(0, \infty)$; therefore

$$
S_{H, L}=\frac{S}{C(N, \mu)^{1 / 2_{\mu}^{*}}},
$$

where

$$
S=\inf _{u \in H_{0}^{1}(\Omega) \backslash\{0\}}\left\{\frac{\int_{\Omega}|\nabla u|^{2}}{\left(\int_{\Omega}|u|^{2^{*}}\right)^{2 / 2^{*}}}, u \in H_{0}^{1}(\Omega) \backslash\{0\}\right\} .
$$


For $\varepsilon>0$

$$
U(x)=\frac{\left[N(N-2) \varepsilon^{2}\right]^{(N-2) / 4}}{\left(\varepsilon^{2}+|x|^{2}\right)^{(N-2) / 2}}
$$

is a solution of the problem

$$
-\Delta u=\int_{\mathbb{R}^{N}} \frac{|u|^{2_{\mu}^{*}}}{|x-y|^{\mu}} d y|u|^{2_{\mu}^{*}-2} u, \quad \text { in } \mathbb{R}^{N}
$$

with $\int_{\mathbb{R}^{N}}|\nabla U|^{2} d x=\int_{\mathbb{R}^{N}} \int_{\mathbb{R}^{N}}|U(x)|^{2_{\mu}^{*}}|U(y)|^{2_{\mu}^{*}} /|x-y|^{\mu} d x d y$ $=S_{H, L}^{(2 N-\mu) /(N-\mu+2)}$.

Definition 8. (i) For $\rho \in \mathbb{R}$, if $I_{\lambda, \mu}\left(u_{n}\right)=\rho+o(1), I_{\lambda, \mu}^{\prime}\left(u_{n}\right)=$ $o(1)$ strongly in $H^{-1}(\Omega)$ as $n \rightarrow \infty$, then the sequence $\left\{u_{n}\right\}$ is a $(P S)_{\rho}$ sequence in $H_{0}^{1}(\Omega)$;

(ii) $I_{\lambda, \mu}$ satisfies the $(P S)_{\rho}$ condition $H_{0}^{1}(\Omega)$ if every $(P S)_{\rho}$ sequence in $H_{0}^{1}(\Omega)$ for $I_{\lambda, \mu}$ has a convergent subsequence.

Though $I_{\lambda, \mu}$ is not bounded below on $H_{0}^{1}(\Omega)$, we can construct the following Nehari manifold:

$$
\begin{aligned}
& \mathscr{M}_{\lambda, \mu}=\left\{u \in H_{0}^{1}(\Omega) \backslash\{0\} \mid\left\langle I_{\lambda, \mu}^{\prime}(u), u\right\rangle=0\right\} \\
& =\left\{u \in H_{0}^{1}(\Omega) \backslash\{0\} \mid\|u\|^{2}\right. \\
& \quad-\int_{\Omega} \int_{\Omega} g(x) \frac{|u(x)|^{2_{\mu}^{*}}|u(y)|^{2_{\mu}^{*}}}{|x-y|^{\mu}} d x d y \\
& \left.\quad-\int_{\Omega} f_{\lambda}(x)|u|^{q}=0\right\} .
\end{aligned}
$$

Define the fiber map $\Phi_{\lambda, \mu}: \mathbb{R}^{+} \rightarrow \mathbb{R}$ by $\Phi_{\lambda, \mu}(t)=I_{\lambda, \mu}(t u)$.

Lemma 9. $I_{\lambda, \mu}(u)$ is coercive and bounded below on $\mathscr{M}_{\lambda, \mu}$.

Proof. If $u \in \mathscr{M}_{\lambda, \mu}$, by (14) we have

$$
\begin{aligned}
& I_{\lambda, \mu}(u)=\frac{1}{2}\|u\|^{2}-\frac{1}{22_{\mu}^{*}} \\
& \quad \int_{\Omega} \int_{\Omega} g(x) \frac{|u(x)|^{2_{\mu}^{*}}|u(y)|^{2_{\mu}^{*}}}{|x-y|^{\mu}} d x d y \\
& \quad-\int_{\Omega} f_{\lambda}(x)|u|^{q}=\left(\frac{1}{2}-\frac{1}{22_{\mu}^{*}}\right)\|u\|^{2} \\
& \quad-\left(\frac{1}{q}-\frac{1}{22_{\mu}^{*}}\right) \int_{\Omega} f_{\lambda}|u|^{q}=\left(\frac{1}{2}-\frac{1}{22_{\mu}^{*}}\right)\|u\|^{2}
\end{aligned}
$$

$$
\begin{aligned}
& -\left(\frac{1}{q}-\frac{1}{22_{\mu}^{*}}\right) \int_{\Omega}\left(\lambda f_{+}+f_{-}\right)|u|^{q} \geq\left(\frac{1}{2}-\frac{1}{22_{\mu}^{*}}\right) \\
& \cdot\|u\|^{2}-\left(\frac{1}{q}-\frac{1}{22_{\mu}^{*}}\right) \lambda\left\|f_{+}\right\|_{\infty} S^{-q / 2}|\Omega|^{\left(2^{*}-q\right) / 2 *}\|u\|^{q} \\
& \geq\left(\frac{1}{2}-\frac{1}{22_{\mu}^{*}}\right)\|u\|^{2}-\left(\frac{1}{q}-\frac{1}{22_{\mu}^{*}}\right) \lambda\left\|f_{+}\right\|_{\infty}|\Omega|^{1 / 22^{*}} \\
& \cdot\left(S_{H, L} C(N, \mu)^{1 / 2_{\mu}^{*}}\right)^{-q / 2}\|u\|^{q},
\end{aligned}
$$

so $I_{\lambda, \mu}(u)$ is coercive and bounded below on $\mathscr{M}_{\lambda, \mu}$. This lemma is completed.

Lemma 10. Assume that $u_{1}$ is a local minimizer for $I_{\lambda, \mu}$ on $\mathscr{M}_{\lambda, \mu}$ and $u_{1} \notin \mathscr{M}_{\lambda, \mu}^{0}$. Then $I_{\lambda, \mu}^{\prime}\left(u_{1}\right)=0$. Moreover, if $u_{1}$ is a nontrivial nonnegative function in $\Omega$, then $u_{1}$ is a positive solution of (1).

Proof. Since $u_{1}$ is a local minimizer for $I_{\lambda, \mu}$ on $\mathscr{M}_{\lambda, \mu}, u_{1}$ is a solution of the optimization problem

$$
\begin{array}{ll}
\text { minimize } & I_{\lambda, \mu}(u) \\
\text { subject to } & \Gamma(u)=0,
\end{array}
$$

where

$$
\begin{aligned}
\Gamma(u)= & \int_{\Omega}|\nabla u|^{2} \\
& -\int_{\Omega} \int_{\Omega} g(x) \frac{|u(x)|^{2_{\mu}^{*}}|u(y)|^{2_{\mu}^{*}}}{|x-y|^{\mu}} d x d y \\
& -\int_{\Omega} f_{\lambda}(x)|u|^{q} .
\end{aligned}
$$

Hence, using Lagrange multipliers, there exists $\xi \in \mathbb{R}$, such that $I_{\lambda, \mu}^{\prime}\left(u_{1}\right)=\xi \Gamma^{\prime}\left(u_{1}\right)$, which implies

$$
\left\langle I_{\lambda, \mu}^{\prime}\left(u_{1}\right), u_{1}\right\rangle=\xi\left\langle\Gamma^{\prime}\left(u_{1}\right), u_{1}\right\rangle \text {. }
$$

Since $u_{1} \in \mathscr{M}_{\lambda, \mu},\left\langle I_{\lambda, \mu}^{\prime}\left(u_{1}\right), u_{1}\right\rangle=0$ and $\int_{\Omega}\left|\nabla u_{1}\right|^{2}-$ $\int_{\Omega} \int_{\Omega} g(x)\left(\left|u_{1}(x)\right|^{2_{\mu}^{*}}\left|u_{1}(y)\right|^{2_{\mu}^{*}} /|x-y|^{\mu}\right) d x d y-\int_{\Omega} f_{\lambda}(x)\left|u_{1}\right|^{q}$,
one has

$$
\begin{aligned}
\langle & \left.\Gamma^{\prime}\left(u_{1}\right), u_{1}\right\rangle \\
= & (2-q)\left\|\nabla u_{1}\right\|^{2} \\
& -\left(22_{\mu}^{*}-q\right) \int_{\Omega} \int_{\Omega} g(x) \frac{\left|u_{1}(x)\right|^{2_{\mu}^{*}}\left|u_{1}(y)\right|^{2_{\mu}^{*}}}{|x-y|^{\mu}} d x d y .
\end{aligned}
$$

Hence, if $u_{1} \notin \mathscr{M}_{\lambda, \mu},\left\langle\Gamma^{\prime}\left(u_{1}\right), u_{1}\right\rangle \neq 0$, combining (21) $\xi=0$, then $I_{\lambda, \mu}(u)$ in $H_{0}^{1}(\Omega)$. Using Harnack inequality, thus, $u_{1}$ is a positive solution of (1) in $\Omega$. The lemma is completed. 
From $\Phi_{\lambda, \mu}(t)=I_{\lambda, \mu}(t u)$, we obtain that

$$
\begin{aligned}
\Phi_{\lambda, \mu}( & t) \\
= & \frac{1}{2}\|t u\|^{2} \\
& \quad-\frac{1}{22_{\mu}^{*}} \int_{\Omega} \int_{\Omega} g(x) \frac{|t u(x)|^{2_{\mu}^{*}}|t u(y)|^{2_{\mu}^{*}}}{|x-y|^{\mu}} d x d y \\
& -\int_{\Omega} f_{\lambda}(x)|t u|^{q},
\end{aligned}
$$

for $t>0, u \in H_{0}^{1}(\Omega)$, we get

$$
\begin{aligned}
& \Phi_{\lambda, \mu}^{\prime}(t)=t\|u\|^{2} \\
& -t^{22_{\mu}^{*}-1} \int_{\Omega} \int_{\Omega} g(x) \frac{|u(x)|^{2_{\mu}^{*}}|u(y)|^{2_{\mu}^{*}}}{|x-y|^{\mu}} d x d y \\
& -t^{q-1} \int_{\Omega} f_{\lambda}(x)|u|^{q}, \\
& \Phi_{\lambda, \mu}^{\prime \prime}(t)=\|u\|^{2}-\left(22_{\mu}^{*}-1\right) \\
& \cdot t^{22_{\mu}^{*}-2} \int_{\Omega} \int_{\Omega} g(x) \frac{|u(x)|^{2_{\mu}^{*}}|u(y)|^{2_{\mu}^{*}}}{|x-y|^{\mu}} d x d y \\
& -(q-1) t^{q-2} \int_{\Omega} f_{\lambda}(x)|u|^{q} .
\end{aligned}
$$

Obviously, $\Phi_{\lambda, \mu}^{\prime}(1)=0$; we divide $\mathscr{M}_{\lambda, \mu}$ into the following three sets

$$
\begin{aligned}
& \mathscr{M}_{\lambda, \mu}^{+}=\left\{u \in \mathscr{M}_{\lambda, \mu} \mid \Phi_{\lambda, \mu}^{\prime \prime}(1)>0\right\} ; \\
& \mathscr{M}_{\lambda, \mu}^{0}=\left\{u \in \mathscr{M}_{\lambda, \mu} \mid \Phi_{\lambda, \mu}^{\prime \prime}(1)=0\right\} ; \\
& \mathscr{M}_{\lambda, \mu}^{-}=\left\{u \in \mathscr{M}_{\lambda, \mu} \mid \Phi_{\lambda, \mu}^{\prime \prime}(1)<0\right\} .
\end{aligned}
$$

For any $u \in \mathscr{M}_{\lambda, \mu}$, one has

$$
\begin{aligned}
\Phi_{\lambda, \mu}^{\prime \prime}(1) & \|u\|^{2} \\
& -\left(22_{\mu}^{*}-1\right) \int_{\Omega} \int_{\Omega} g(x) \frac{|u(x)|^{2_{\mu}^{*}}|u(y)|^{2_{\mu}^{*}}}{|x-y|^{\mu}} d x d y \\
& -(q-1) \int_{\Omega} f_{\lambda}(x)|u|^{q} \\
= & \left(2-22_{\mu}^{*}\right)\|u\|^{2}-\left(q-22_{\mu}^{*}\right) \int_{\Omega} f_{\lambda}|u|^{q} \\
= & (2-q)\|u\|^{2} \\
& -\left(22_{\mu}^{*}-q\right) \int_{\Omega} \int_{\Omega} g(x) \frac{|u(x)|^{2_{\mu}^{*}}|u(y)|^{2_{\mu}^{*}}}{|x-y|^{\mu}} d x d y .
\end{aligned}
$$

Therefore, we can derive
Lemma 11. (i) For every $u \in \mathscr{M}_{\lambda, \mu}^{+} \cup \mathscr{M}_{\lambda, \mu}^{0}$, then $\int_{\Omega} f_{\lambda}(x)|u|^{q}>$ 0 .

(ii) For every $u \in \mathscr{M}_{\lambda, \mu}^{-} \cup \mathscr{M}_{\lambda, \mu}^{0}$, then $\int_{\Omega} \int_{\Omega} g(x)\left(|u(x)|^{2_{\mu}^{*}}|u(y)|^{2_{\mu}^{*}} /|x-y|^{\mu}\right) d x d y>0$.

Lemma 12. For $\lambda \in\left(0, \lambda_{*}\right)$, then $\mathscr{M}_{\lambda, \mu}^{0}=\emptyset$.

Proof. Assuming the contrary, there is $\lambda \in\left(0, \lambda_{*}\right)$ such that $\mathscr{M}_{\lambda, \mu}^{0} \neq \emptyset$, then for $u \in \mathscr{M}_{\lambda, \mu}^{0}$, by (26) and Proposition 6, which implies

$$
\begin{aligned}
\|u\|^{2} & =\frac{22_{\mu}^{*}-q}{2-q} \int_{\Omega} \int_{\Omega} g(x) \frac{|u(x)|^{2_{\mu}^{*}}|u(y)|^{2_{\mu}^{*}}}{|x-y|^{\mu}} d x d y \\
& \leq \frac{22_{\mu}^{*}-q}{2-q}|u|_{2^{*}}^{2.2_{\mu}^{*}} S_{H, L}^{-2_{\mu}^{*}}\left|g_{+}\right|_{\infty},
\end{aligned}
$$

so

$$
\|u\| \geq\left(\frac{2-q}{22_{\mu}^{*}-q} S_{H, L}^{2_{\mu}^{*}}\left|g_{+}\right|_{\infty}^{-1}\right)^{1 /\left(22_{\mu}^{*}-2\right)} .
$$

Similarly, coupling with (26) and (14), which infers that

$$
\begin{gathered}
\|u\|^{2}=\frac{q-22_{\mu}^{*}}{2-22_{\mu}^{*}} \int_{\Omega} f_{\lambda}(x)|u|^{q} \leq \frac{q-22_{\mu}^{*}}{2-22_{\mu}^{*}} \lambda\left\|f_{+}\right\|_{\infty} \\
\cdot\left(S_{H, L} C(N, \mu)^{1 / 2_{\mu}^{*}}\right)^{-q / 2}\|u\|^{q}=\frac{q-22_{\mu}^{*}}{2-22_{\mu}^{*}} \\
\cdot \lambda|\Omega|^{\left(2^{*}-q\right) / 2^{*}}\left\|f_{+}\right\|_{\infty}\left(S_{H, L} C(N, \mu)^{1 / 2_{\mu}^{*}}\right)^{-q / 2}\|u\|^{q},
\end{gathered}
$$

hence

$$
\begin{gathered}
\|u\| \leq\left[\frac{q-22_{\mu}^{*}}{2-22_{\mu}^{*}} \lambda|\Omega|^{\left(2^{*}-q\right) / 2^{*}}\left\|f_{+}\right\|_{\infty}\right. \\
\left.\cdot\left(S_{H, L} C(N, \mu)^{1 / 2_{\mu}^{*}}\right)^{-q / 2}\right]^{1 /(2-q)} .
\end{gathered}
$$

From the above inequalities, we deduce that

$$
\begin{gathered}
\lambda \geq\left(\frac{2-q}{22_{\mu}^{*}-q}\left|g_{+}\right|_{\infty}^{-1}\right)^{(2-q) /\left(22_{\mu}^{*}-q\right)}\left(\frac{22_{\mu}^{*}-2}{22_{\mu}^{*}-q}\left|f_{+}\right|_{\infty}^{-1}\right) \\
\cdot|\Omega|^{\left(q-2^{*}\right) / 2^{*}} C(N, \mu)^{q / 22_{\mu}^{*}} S_{H, L}^{2_{\mu}^{*}(2-q) / 22_{\mu}^{*}+q / 2}:=\lambda_{*},
\end{gathered}
$$

which is a contradiction.

Letting $u \in \Phi_{\lambda, \mu}(t)$, one has

$$
\begin{aligned}
& \frac{d}{d t} I_{\lambda, \mu}(t u)=t\|u\|^{2} \\
& \quad-t^{22_{\mu}^{*}-1} \int_{\Omega} \int_{\Omega} g(x) \frac{|u(x)|^{2_{\mu}^{*}}|u(y)|^{2_{\mu}^{*}}}{|x-y|^{\mu}} d x d y \\
& \quad-t^{q-1} \int_{\Omega} f_{\lambda}(x)|u|^{q}=t^{q-1}\left(t^{2-q}\|u\|^{2}\right.
\end{aligned}
$$




$$
\begin{aligned}
& -t^{22_{\mu}^{*}-q} \int_{\Omega} \int_{\Omega} g(x) \frac{|u(x)|^{2_{\mu}^{*}}|u(y)|^{2_{\mu}^{*}}}{|x-y|^{\mu}} d x d y \\
& \left.-\int_{\Omega} f_{\lambda}(x)|u|^{q}\right) .
\end{aligned}
$$

Let

$$
\begin{aligned}
\varphi(t)= & t^{2-q}\|u\|^{2} \\
& -t^{22_{\mu}^{*}-q} \int_{\Omega} \int_{\Omega} g(x) \frac{|u(x)|^{2_{\mu}^{*}}|u(y)|^{2_{\mu}^{*}}}{|x-y|^{\mu}} d x d y .
\end{aligned}
$$

Assume $t u \in \mathscr{M}_{\lambda, \mu}$ if and only if $\varphi(t)=\int_{\Omega} f_{\lambda}(x)|u|^{q}$. So

$$
\begin{aligned}
& \varphi^{\prime}(t)=(2-q) t^{1-q}\|u\|^{2}-\left(22_{\mu}^{*}-q\right) \\
& \cdot t^{22_{\mu}^{*}-q-1} \int_{\Omega} \int_{\Omega} g(x) \frac{|u(x)|^{2_{\mu}^{*}}|u(y)|^{2_{\mu}^{*}}}{|x-y|^{\mu}} d x d y .
\end{aligned}
$$

Clearly, $t u \in \mathscr{M}_{\lambda, \mu}$, then $t^{q-1} \varphi^{\prime}(1)=\Phi_{\lambda, \mu}^{\prime \prime}(1)$. Hence, $t u \in$ $\mathscr{M}_{\lambda, \mu}^{+}$(or $\mathscr{M}_{\lambda, \mu}^{-}$) provided $\varphi^{\prime}(t)>0$ (or $\left.<0\right)$ ). $\varphi(t)$ can attain its maximum at $t=t_{\max }$ duo to $\lim _{t \rightarrow \infty} \varphi(t)=\infty$, where

$$
\begin{aligned}
& t_{\max }=\left[\frac{(2-q)\|u\|^{q}}{\left(22_{\mu}^{*}-q\right) \int_{\Omega} \int_{\Omega} g(x)\left(|u(x)|^{2_{\mu}^{*}}|u(y)|^{2_{\mu}^{*}} /|x-y|^{\mu}\right) d x d y}\right]^{1 /\left(22_{\mu}^{*}-2\right)}>0, \\
& \varphi\left(t_{\max }\right) \\
& =\left[\frac{(2-q)\|u\|^{q}}{\left(22_{\mu}^{*}-q\right) \int_{\Omega} \int_{\Omega} g(x)\left(|u(x)|^{2_{\mu}^{*}}|u(y)|^{2_{\mu}^{*}} /|x-y|^{\mu}\right) d x d y}\right]^{(2-q) /\left(22_{\mu}^{*}-2\right)}\|u\|^{2} \\
& -\left[\frac{(2-q)\|u\|^{q}}{\left(22_{\mu}^{*}-q\right) \int_{\Omega} \int_{\Omega} g(x)\left(|u(x)|^{2_{\mu}^{*}}|u(y)|^{2_{\mu}^{*}} /|x-y|^{\mu}\right) d x d y}\right]^{\left(22_{\mu}^{*}-q\right) /\left(22_{\mu}^{*}-2\right)} \int_{\Omega} \int_{\Omega} g(x) \frac{|u(x)|^{2_{\mu}^{*}}|u(y)|^{2_{\mu}^{*}}}{|x-y|^{\mu}} d x d y \\
& =\left[\frac{(2-q)\|u\|^{q}}{\left(22_{\mu}^{*}-q\right) \int_{\Omega} \int_{\Omega} g(x)\left(|u(x)|^{2 *}|u(y)|^{2_{\mu}^{*}} /|x-y|^{\mu}\right) d x d y}\right]^{\left(22_{\mu}^{*}-q\right) /\left(22_{\mu}^{*}-2\right)}\left(\|u\|^{2}-\frac{2-q}{22_{\mu}^{*}-q}\|u\|^{q}\right) \\
& =\left(\frac{2-q}{22_{\mu}^{*}-q}\right)^{\left(22_{\mu}^{*}-q\right) /\left(22_{\mu}^{*}-2\right)}\left(\frac{22_{\mu}^{*}-2}{22_{\mu}^{*}-q}\right)\|u\|^{q} S_{H, L}^{2_{\mu}^{*}\left(\left(22_{\mu}^{*}-2\right) /\left(22_{\mu}^{*}-q\right)\right)}\|u\|^{-22_{\mu}^{*}} \\
& \geq\left\|f_{+}\right\|_{\infty}^{-1}\left(\frac{2-q}{22_{\mu}^{*}-q}\right)^{\left(22_{\mu}^{*}-q\right) /\left(22_{\mu}^{*}-2\right)}\left(\frac{22_{\mu}^{*}-2}{22_{\mu}^{*}-q}\right) S_{H, L}^{2_{\mu}^{*}\left(\left(22_{\mu}^{*}-2\right) /\left(22_{\mu}^{*}-q\right)\right)}\|u\|^{-22_{\mu}^{*}} \int_{\Omega} f_{\lambda}(x)|u|^{q}>\tilde{\lambda} \int_{\Omega} f_{\lambda}(x)|u|^{q},
\end{aligned}
$$

where $\tilde{\lambda}=\left\|f_{+}\right\|_{\infty}^{-1}\left((2-q) /\left(22_{\mu}^{*}-q\right)\right)^{\left(22_{\mu}^{*}-q\right) /\left(22_{\mu}^{*}-2\right)}\left(\left(22_{\mu}^{*}-\right.\right.$ 2)/(22* $-q)) S_{H, L}^{2_{\mu}^{*}\left(\left(22_{\mu}^{*}-2\right) /\left(22_{\mu}^{*}-q\right)\right)}\|u\|^{-22_{\mu}^{*}}$.

Lemma 13. For every $u \in H_{0}^{1}(\Omega)$, it follows that

(i) if $\int_{\Omega} f_{\lambda}(x)|u|^{q} \leq 0, \lambda>0$, there exists a unique $t^{-}=$ $t^{-}(u)>t_{\max }(u)$ such that $t^{-} u \in \mathscr{M}_{\lambda, \mu^{2}}^{-}$and $\Phi_{\lambda, \mu}$ is increasing on $\left(0, t^{-}\right)$, decreasing on $\left(t^{-}, \infty\right)$.

Moreover

$$
I_{\lambda, \mu}\left(t^{-} u\right)=\sup _{t \geq 0} I_{\lambda, \mu}(t u)
$$

(ii)if $\int_{\Omega} f_{\lambda}(x)|u|^{q}>0$, then there exist unique $0<t^{+}=t^{+}(u)<$ $t_{\max }<t^{-}=t^{-}(u)$ such that $t^{-}(u) u \in \mathscr{M}_{\lambda, \mu}^{-}, t^{+}(u) u \in \mathscr{M}_{\lambda, \mu}^{+}$ and $\Phi_{\lambda, \mu}$ is increasing on $\left(t^{+}, t^{-}\right)$, decreasing on $\left(t^{-},+\infty\right) \cup$ $\left(0, t^{+}\right)$. Moreover

$$
\begin{aligned}
& I_{\lambda, \mu}\left(t^{-} u\right)=\sup _{t \geq t_{\max }} I_{\lambda, \mu}(t u), \\
& I_{\lambda, \mu}\left(t^{+} u\right)=\inf _{0 \leq t \leq t_{\max }} I_{\lambda, \mu}(t u) .
\end{aligned}
$$

Proof. (i)Assume that $\int_{\Omega} f_{\lambda}(x)|u|^{q} \leq 0$. There exists a solution $t^{-}\left(t^{-} \geq t_{\max }\right)$, with $\varphi=\int_{\Omega} f_{\lambda}(x)|u|^{q}$, then $\varphi_{t^{-}}^{\prime}<0$, and $\Phi_{\lambda, \mu}^{\prime}\left(t^{-}\right)=0$. Consequently, $\Phi_{\lambda, \mu}$ has a unique critical point at $t=t^{-}$and $\varphi_{t^{-}}^{\prime \prime}<0$. Since $t^{q-1} \varphi^{\prime}(1)=\Phi_{\lambda, \mu}^{\prime \prime}(1)$. Hence, (36) holds and $t^{-} u \in \mathscr{M}_{\lambda, \mu}^{-}$. 
(ii) Assume $\int_{\Omega} f_{\lambda}(x)|u|^{q}>0$, then $\varphi_{t_{\max }}(u)>$ $\int_{\Omega} f_{\lambda}(x)|u|^{q}$. There are two solutions $t^{-}, t^{+},\left(t^{+}<t_{\max }<t^{-}\right)$, satisfying $\varphi(t)=\int_{\Omega} f_{\lambda}(x)|u|^{q}$, such that $\varphi^{\prime}\left(t^{-}\right)<0, \varphi^{\prime}\left(t^{+}\right)>$ 0 , which means that these two solutions of $u$ depend on $\mathscr{M}_{\lambda, \mu}$, where $t^{-} u \in \mathscr{M}_{\lambda, \mu}^{-}, t_{2} u \in \mathscr{M}_{\lambda . \mu}^{+}$. So according to $t^{q-1} \varphi^{\prime}(1)=$ $\Phi_{\lambda, \mu}^{\prime \prime}(1), \Phi_{\lambda, \mu}$ has critical points at $t^{-}=t^{+}, \Phi_{\lambda, \mu}\left(t^{-}\right)<$ $0, \Phi_{\lambda, \mu}\left(t^{+}\right)>0$. Thus, $\Phi_{\lambda, \mu}$ is increasing on $\left(t^{+}, t^{-}\right)$, and $\left(0, t^{+}\right) \cup\left(t^{+},+\infty\right)$, (37) holds.

This lemma is completed.

Furthermore, we have

$$
\mathscr{M}_{\lambda, \mu}=\mathscr{M}_{\lambda, \mu}^{+} \cup \mathscr{M}_{\lambda, \mu}^{-} .
$$

Define

$$
\begin{aligned}
& \alpha_{\lambda, \mu}=\inf _{u \in \mathscr{M}_{\lambda, \mu}} I_{\lambda, \mu}(u), \\
& \alpha_{\lambda, \mu}^{+}=\inf _{u \in \mathscr{M}_{\lambda, \mu}^{+}} I_{\lambda, \mu}(u), \\
& \alpha_{\lambda, \mu}^{-}=\inf _{u \in \mathscr{M}_{\lambda, \mu}^{-}} I_{\lambda, \mu}(u) .
\end{aligned}
$$

Theorem 14. (i) $\alpha_{\lambda, \mu}^{+}<0$ for all $\lambda \in\left(0, \lambda_{*}\right)$.

(ii) If $\lambda \in\left(0,(q / 2) \lambda_{*}\right)$, then there exists a positive number $d_{1}$, such that $\alpha_{\lambda, \mu}^{-}>d_{1}$.

Especially, $\alpha_{\lambda, \mu}^{+}=\inf _{u \in M_{\lambda, \mu}} I_{\lambda, \mu}(u)$ for all $\lambda \in\left(0,(q / 2) \lambda_{*}\right)$.

Proof. (i) Supposing that $u \in \mathscr{M}_{\lambda, \mu}^{+}$from (26), one has

$$
\|u\|^{2}<\frac{q-22_{\mu}^{*}}{2-22_{\mu}^{*}} \int_{\Omega} f_{\lambda}(x)|u|^{q},
$$

by Lemma 9, we derive

$$
\begin{aligned}
I_{\lambda, \mu}(u)= & \left(\frac{1}{2}-\frac{1}{22_{\mu}^{*}}\right)\|u\|^{2} \\
& -\left(\frac{1}{q}-\frac{1}{22_{\mu}^{*}}\right) \int_{\Omega} f_{\lambda}(x)|u|^{q} \\
\leq & \left(\frac{1}{2}-\frac{1}{22_{\mu}^{*}}\right) \frac{q-22_{\mu}^{*}}{2-22_{\mu}^{*}} \int_{\Omega} f_{\lambda}(x)|u|^{q} \\
& -\left(\frac{1}{q}-\frac{1}{22_{\mu}^{*}}\right) \int_{\Omega} f_{\lambda}(x)|u|^{q} \\
= & \frac{q-2}{2 q} \int_{\Omega} f_{\lambda}(x)|u|^{q}<0,
\end{aligned}
$$

Thus, $\alpha_{\lambda, \mu}^{+}<0$. get

(ii) Suppose that $u \in \mathscr{M}_{\lambda, \mu}^{-}$from (11), (26), and ( $\left.g_{1}\right)$, we

$$
\|u\|^{2} \leq\left(\frac{2-q}{22_{\mu}^{*}} S_{H, L}^{2_{\mu}^{*}}\left|g_{+}\right|_{\infty}^{-1}\right)^{1 /\left(22_{\mu}^{*}-2\right)}, \quad \forall u \in \mathscr{M}_{\lambda, \mu}^{-} .
$$

Moreover

$$
\begin{aligned}
I_{\lambda, \mu} & (u) \geq\left(\frac{1}{2}-\frac{1}{22_{\mu}^{*}}\right)\|u\|^{2}-\left(\frac{1}{q}-\frac{1}{22_{\mu}^{*}}\right) \\
\cdot & \lambda|\Omega|^{\left(2^{*}-q\right) / 2^{*}}\left\|f_{+}\right\|_{\infty}\left(S_{H, L} C(N, \mu)^{1 / 2_{\mu}^{*}}\right)^{-q / 2}\|u\|^{q} \\
= & \|u\|^{q}\left[\frac{2_{\mu}^{*}-1}{22_{\mu}^{*}}\|u\|^{2-q}-\frac{22_{\mu}^{*}-q}{22_{\mu}^{*} q} \lambda|\Omega|^{\left(2^{*}-q\right) / 2^{*}}\right. \\
\cdot & \left.\left\|f_{+}\right\|_{\infty}\left(S_{H, L} C(N, \mu)^{1 / 2_{\mu}^{*}}\right)^{-q / 2}\right] \geq\left(\frac{2-q}{22_{\mu}^{*}-q}\right. \\
\cdot & \left.S_{H, L}^{2_{\mu}^{*}}\left|g_{+}\right|_{\infty}^{-1}\right)^{q /\left(22_{\mu}^{*}-2\right)} \\
\cdot & {\left[\frac{2_{\mu}^{*}-1}{22_{\mu}^{*}}\left(\frac{2-q}{22_{\mu}^{*}-q} S_{H, L}^{2_{\mu}^{*}}\left|g_{+}\right|_{\infty}^{-1}\right)^{(2-q) /\left(22_{\mu}^{*}-2\right)}\right.} \\
& -\frac{22_{\mu}^{*}-q}{22_{\mu}^{*} q} \lambda|\Omega|^{\left(2^{*}-q\right) / 2^{*}}\left|f_{+}\right|_{\infty} \\
& \left.\cdot\left(S_{H, L} C(N, \mu)^{1 / 2_{\mu}^{*}}\right)^{-q / 2}\right] .
\end{aligned}
$$

Hence, if $\lambda \in\left(0,(q / 2) \lambda_{*}\right)$, such that

$$
I_{\lambda, \mu}(u)>d_{1}, \quad \forall u \in \mathscr{M}_{\lambda, \mu}^{-}
$$

where $d_{1}$ depends on $\lambda, \mu, s, q, N, S_{H, L},|\Omega|,\left|f_{+}\right|_{\infty},\left|g_{+}\right|_{\infty}$.

This completes the proof.

Lemma 15. Every sequence $\left\{u_{n}\right\} \in H_{0}^{1}(\Omega)$ that satisfies $I_{\lambda, \mu}\left(u_{n}\right)=\beta_{1}+o(1)$ with $\beta_{1}<\alpha_{\lambda, \mu}^{+}+S_{H, L}^{N / 2} C(N, \mu)^{N / 22_{\mu}^{*}}$ and $I_{\lambda, \mu}^{\prime}\left(u_{n}\right)=o(1)$ in $H^{-1}(\Omega)$ has a convergent subsequence.

Proof. For $\left\{u_{n}\right\}$, there exists $u_{0} \geq 0$ such that

$$
\begin{aligned}
u_{n} & \rightarrow u_{0}, \quad \text { in } H_{0}^{1}(\Omega), \\
u_{n} & \longrightarrow u_{0}, \quad \text { in } L^{p}(\Omega), 1 \leq p<2^{*}, \\
u_{n}(x) & \longrightarrow u_{0}(x), \quad \text { a.e. in } \Omega .
\end{aligned}
$$

Therefore

$$
\begin{aligned}
& \left\langle I_{\lambda, \mu}^{\prime}\left(u_{0}\right), u_{0}\right\rangle \\
& =\left\|u_{0}\right\|^{2}-\int_{\mathbb{R}^{N}} \int_{\mathbb{R}^{N}} \frac{\left|u_{0}(x)\right|^{2_{\mu}^{*}}\left|u_{0}(y)\right|^{2_{\mu}^{*}}}{|x-y|^{\mu}} d x d y \\
& \quad-\int_{\Omega} f_{\lambda}(x)\left|u_{0}\right|^{q}=0, \\
& \int_{\Omega} f_{\lambda}(x)\left|u_{n}\right|^{q}=\int_{\Omega} f_{\lambda}(x)\left|u_{0}\right|^{q}+o(1) .
\end{aligned}
$$


So, by Theorem $14, I_{\lambda, \mu}\left(u_{0}\right) \geq \alpha_{\lambda, \mu}^{+}$, we know that

$$
\begin{aligned}
\int_{\Omega}\left|\nabla\left(u_{n}\right)\right|^{2}= & \int_{\Omega}\left|\nabla\left(u_{0}\right)\right|^{2}+\int_{\Omega}\left|\nabla\left(u_{n}-u_{0}\right)\right|^{2} \\
& +o(1) .
\end{aligned}
$$

Thus

$$
\begin{aligned}
I_{\lambda, \mu}\left(u_{n}\right)= & I_{\lambda, \mu}\left(u_{0}\right)+I_{\lambda, \mu}\left(u_{n}-u_{0}\right)+o(1), \\
o(1)= & \left\langle u_{n}-u_{0}, I_{\lambda, \mu}\left(u_{n}\right)\right\rangle \\
= & \left\langle u_{n}-u_{0}, I_{\lambda, \mu}\left(u_{n}\right)-I_{\lambda, \mu}\left(u_{0}\right)\right\rangle \\
= & \left\|u_{n}-u_{0}\right\|^{2} \\
& -\int_{\Omega} \int_{\Omega} \frac{\left|u_{0}(x)\right|^{2_{\mu}^{*}}\left|u_{0}(y)\right|^{2_{\mu}^{*}}}{|x-y|^{\mu}} d x d y,
\end{aligned}
$$

which implies

$$
\begin{aligned}
& \frac{4-\mu}{2(2 N-\mu)}\left\|u_{n}-u_{0}\right\|^{2}=I_{\lambda, \mu}\left(u_{n}-u_{0}\right) \\
& \quad=I_{\lambda, \mu}\left(u_{n}\right)-I_{\lambda, \mu}\left(u_{0}\right)+o(1) \\
& \quad \leq I_{\lambda, \mu}\left(u_{n}\right)-\alpha_{\lambda, \mu}^{+}+o(1) .
\end{aligned}
$$

Furthermore

$$
\left\|u_{n}-u_{0}\right\|^{2}<S_{H, L}^{N / 2} C(N, \mu)^{N / 22_{\mu}^{*}} .
$$

Lemma 16. For every $\lambda \in\left(0, \lambda_{*}\right)$, then $I_{\lambda, \mu}$ has a minimizer $u_{1}$ in $\mathscr{M}_{\lambda, \mu}^{+}$, satisfying that

(i) $I_{\lambda, \mu}\left(u_{1}\right)=\alpha_{\lambda, \mu}^{+}$;

(ii) $u_{1}$ is a positive solution of (1);

(iii) $I_{\lambda, \mu}\left(u_{1}\right) \rightarrow 0$ as $\lambda \rightarrow 0$.

Proof. (i)From Lemma 15, there exists a minimizing sequence $\left\{u_{n}\right\}$ for $I_{\lambda, \mu}$, such that

$$
\begin{aligned}
& I_{\lambda, \mu}\left(u_{n}\right)=\beta+o(1), \\
& I_{\lambda, \mu}^{\prime}\left(u_{n}\right)=o(1),
\end{aligned}
$$

$$
\text { in } H_{0}^{1}(\Omega) \text {. }
$$

Noting that $I_{\lambda, \mu}$ is coercive and bounded on $\mathscr{M}_{\lambda, \mu}$ in $H_{0}^{1}(\Omega)$. Going if necessary to a subsequence, we can assume that there exists $u_{1} \in H_{0}^{1}(\Omega)$ such that

$$
\begin{gathered}
u_{n} \rightarrow u_{1}, \quad \text { in } H_{0}^{1}(\Omega), \\
u_{n} \longrightarrow u_{1}, \quad \text { in } L^{p}(\Omega), 1 \leq p<2_{\mu}^{*}, \\
u_{n}(x) \longrightarrow u_{1}(x), \quad \text { a.e. in } \Omega .
\end{gathered}
$$

We show that $u_{1}$ is a solution of (1). $u_{n} \in \mathscr{M}_{\lambda, \mu}$, which derives that

$$
\begin{aligned}
& I_{\lambda, \mu}\left(u_{n}\right) \\
&=\frac{1}{2}\left\|u_{n}\right\|^{2} \\
& \quad-\frac{1}{22_{\mu}^{*}} \int_{\Omega} \int_{\Omega} g(x) \frac{\left|u_{n}(x)\right|^{2_{\mu}^{*}}\left|u_{n}(y)\right|^{2_{\mu}^{*}}}{|x-y|^{\mu}} d x d y \\
& \quad-\int_{\Omega} f_{\lambda}(x)\left|u_{n}\right|^{q} \\
&=\left(\frac{1}{2}-\frac{1}{22_{\mu}^{*}}\right)\left\|u_{n}\right\|^{2}-\left(\frac{1}{q}-\frac{1}{22_{\mu}^{*}}\right) \int_{\Omega} f_{\lambda}\left|u_{n}\right|^{q} \\
& \geq \frac{2_{\mu}^{*}-1}{22_{\mu}^{*}}\left\|u_{n}\right\|^{2}-\frac{22_{\mu}^{*}-q}{22_{\mu}^{*} q} \lambda \int_{\Omega} f_{+}\left|u_{n}\right|^{q},
\end{aligned}
$$

thus

$$
\begin{aligned}
\lambda \int_{\Omega} f_{+}\left|u_{n}\right|^{q}= & \frac{\left(22_{\mu}^{*}-1\right) q}{22_{\mu}^{*}-q}\left\|u_{n}\right\|^{2} \\
& -\frac{22_{\mu}^{*} q}{22_{\mu}^{*}-q} I_{\lambda, \mu}\left(u_{n}\right) .
\end{aligned}
$$

By Theorem 14, (51), (52), (54), one has

$$
\lambda \int_{\Omega} f_{+}\left|u_{n}\right|^{q} \geq \frac{22_{\mu}^{*} q}{22_{\mu}^{*}-q} I_{\lambda, \mu}>0
$$

combining Fatou's Lemma, which implies that

$$
\begin{aligned}
& \alpha_{\lambda, \mu}^{+} \leq I_{\lambda, \mu}\left(u_{1}\right)=\frac{2_{\mu}^{*}-1}{22_{\mu}^{*}}\left\|u_{1}\right\|^{2}-\frac{22_{\mu}^{*}-q}{22_{\mu}^{*} q} \int_{\Omega} f_{\lambda}\left|u_{1}\right|^{q} \\
& \quad \leq \liminf _{n \rightarrow \infty}\left(\frac{2_{\mu}^{*}-1}{22_{\mu}^{*}}\left\|u_{n}\right\|^{2}-\frac{22_{\mu}^{*}-q}{22_{\mu}^{*} q} \lambda \int_{\Omega} f\left|u_{n}\right|^{q}\right) \\
& \quad \leq \liminf _{n \rightarrow \infty} I_{\lambda, \mu}\left(u_{n}\right)=\alpha_{\lambda, \mu}^{+} .
\end{aligned}
$$

Clearly,

$$
\begin{gathered}
I_{\lambda, \mu}^{+}\left(u_{1}\right)=\alpha_{\lambda, \mu}, \\
\lim _{n \rightarrow \infty}\left\|u_{n}\right\|^{2}=\left\|u_{1}\right\|^{2} .
\end{gathered}
$$


(ii) Let $w_{n}=u_{n}-u_{1}$. Using Brézis-Lieb Lemma, such that

$$
\left\|w_{n}\right\|^{2}=\left\|u_{n}\right\|^{2}-\left\|u_{1}\right\|^{2}+o(1),
$$

which infers that $u_{n} \rightarrow u_{1}$ in $H_{0}^{1}(\Omega)$ and $u_{1} \in \mathscr{M}_{\lambda, \mu}^{+}$. On the contrary, if $u_{1} \in \mathscr{M}_{\lambda, \mu^{\prime}}^{-}$, from (26), (55), which gets $\int_{\Omega} \int_{\Omega} g(x)\left(\left|u_{1}(x)\right|^{2_{\mu}^{*}}\left|u_{1}(y)\right|^{2_{\mu}^{*}} /|x-y|^{\mu}\right) d x d y>0$ and $\int_{\Omega} f_{\lambda}\left|u_{1}\right|^{q}>0$. Therefore, by Lemma 13, there are unique $t_{1}$ and $t_{2}$ such that $t_{1} u_{1} \in \mathscr{M}_{\lambda, \mu}^{-}, t_{2} u_{1} \in \mathscr{M}_{\lambda, \mu}^{+}$. Moreover, $t_{2}<t_{1}=1$. By simple calculation, one has

$$
\begin{aligned}
& I_{\lambda, \mu}^{\prime}\left(t_{2} u_{1}\right)=0, \\
& I_{\lambda, \mu}^{\prime \prime}\left(t_{2} u_{1}\right)>0 .
\end{aligned}
$$

So there exists $t_{\lambda} \in\left(t_{2}, t_{1}\right)$ such that $I_{\lambda, \mu}\left(t_{2} u_{1}\right)<I_{\lambda, \mu}\left(t_{\lambda} u_{1}\right)$. Coupling with Lemma 13 which indicates that

$$
I_{\lambda, \mu}\left(t_{2} u_{1}\right)<I_{\lambda, \mu}\left(t_{\lambda} u_{1}\right) \leq I_{\lambda, \mu}\left(t_{1} u_{1}\right)=I_{\lambda, \mu}\left(u_{1}\right),
$$

which is a contradiction. Furthermore, $I_{\lambda, \mu}\left(u_{1}\right)=I_{\lambda, \mu}\left(\left|u_{1}\right|\right)$ and $\left|u_{1}\right| \in \mathscr{M}_{\lambda, \mu}^{+}$. From Lemma 10, we claim that $u_{1}$ is a positive solution of (1). Combining the standard elliptic regularity argument and $f, g \in C(\bar{\Omega})$, we can get $u_{2} \in C^{2}(\Omega) \backslash$ $\{0\}$. By Harnack inequality, we have that $u_{1}>0$ in $\Omega \backslash\{0\}$; that is $u_{1}$ is a positive solution of (1).

(iii) By (26), we get

$$
\begin{aligned}
& \left\|u_{1}\right\|^{2}=\frac{q-22_{\mu}^{*}}{2-22_{\mu}^{*}} \int_{\Omega} f_{\lambda}(x)\left|u_{1}\right|^{q} \\
& \quad \leq \frac{q-22_{\mu}^{*}}{2-22_{\mu}^{*}} \lambda\left\|f_{+}\right\|_{\infty}\left(S_{H, L} C(N, \mu)^{1 / 2^{*}}\right)^{-q / 2}\left\|u_{1}\right\|^{q} .
\end{aligned}
$$

Moreover

$$
\left\|u_{1}\right\|^{2-q}=\frac{\left(q-22_{\mu}^{*}\right) \lambda\left\|f_{+}\right\|_{\infty}}{\left(2-22_{\mu}^{*}\right)\left(S_{H, L} C(N, \mu)^{1 / 2_{\mu}^{*}}\right)^{q / 2}} .
$$

Obviously, $I_{\lambda, \mu}\left(u_{1}\right) \rightarrow 0$ as $\lambda \rightarrow 0$. This completes the proof.

Lemma 17. For every $u \in \mathscr{M}_{\lambda, \mu}$, there exist $\varepsilon>0$ and $a$ differentiable function $\zeta=\zeta(w)>0$ with $w \in H_{0}^{1}(\Omega)$ and $\|w\|<\varepsilon$ such that

$$
\begin{aligned}
\zeta(0) & =1, \\
\zeta(w)(u-w) & \in \mathscr{M}_{\lambda, \mu}, \\
\left\langle\zeta^{\prime}(0), w\right\rangle & =\frac{2 \int_{\Omega} \nabla u \nabla w-22_{\mu}^{*} \int_{\Omega} \int_{\Omega}\left(|u(x)|^{2_{\mu}^{*}}|u(y)|^{2_{\mu}^{*}-2} u(y) w(y) /|x-y|^{\mu}\right) d x d y-q \int_{\Omega} f_{\lambda}|u|^{q-2} u w}{(2-q)\|u\|^{2}-\left(22_{\mu}^{*}-q\right) \int_{\Omega} \int_{\Omega}\left(|u(x)|^{2_{\mu}^{*}}|u(y)|^{2_{\mu}^{*}} /|x-y|^{\mu}\right) d x d y} .
\end{aligned}
$$

Proof. $\forall u \in \mathscr{M}_{\lambda, \mu}$; define a function $F: \mathbb{R} \times H_{0}^{1}(\Omega) \rightarrow \mathbb{R}:$

$$
\begin{aligned}
F(\zeta, w)= & \left\langle I_{\lambda, \mu}^{\prime}(\zeta(u-w)), \zeta(u-w)\right\rangle \\
= & \zeta^{2} \int_{\Omega}|\nabla(u-w)|^{2} \\
& -22_{\mu}^{*} \zeta^{22_{\mu}^{*}} \int_{\Omega} \int_{\Omega} \frac{|u(x)|^{2_{\mu}^{*}}|u(y)|^{2_{\mu}^{*}}}{|x-y|^{\mu}} d x d y \\
& -\zeta^{q} \int_{\Omega} f_{\lambda}|u-w|^{q} .
\end{aligned}
$$

$$
\begin{aligned}
= & 2 \int_{\Omega}|\nabla u|^{2}-22_{\mu}^{*} \int_{\Omega} \int_{\Omega} \frac{|u(x)|^{2_{\mu}^{*}}|u(y)|^{2_{\mu}^{*}}}{|x-y|^{\mu}} d x d y \\
& -q \int_{\Omega} f_{\lambda}|u|^{q} \\
= & (2-q)\|u\|^{2} \\
& -\left(22_{\mu}^{*}-q\right) \int_{\Omega} \int_{\Omega} \frac{|u(x)|^{2_{\mu}^{*}}|u(y)|^{2_{\mu}^{*}}}{|x-y|^{\mu}} d x d y \neq 0 .
\end{aligned}
$$

$F(1,0)=\left\langle I_{\lambda, \mu}^{\prime}(u), u\right\rangle=0$, and

$$
\frac{\partial F}{\partial \zeta}(1,0)
$$

$$
\left\langle\zeta^{\prime}(0), w\right\rangle=\frac{2 \int_{\Omega} \nabla u \nabla w-22_{\mu}^{*} \int_{\Omega} \int_{\Omega}|u(x)|^{2_{\mu}^{*}}|u(y)|^{2_{\mu}^{*}-2} u(y) w(y) /|x-y|^{\mu} d x d y-q \int_{\Omega} f_{\lambda}|u|^{q-2} u w}{(2-q)\|u\|^{2}-\left(22_{\mu}^{*}-q\right) \int_{\Omega} \int_{\Omega}\left(|u(x)|^{2_{\mu}^{*}}|u(y)|^{2_{\mu}^{*}} /|x-y|^{\mu}\right) d x d y},
$$

$F(\zeta(w), w)=0$
Applying the implicit function theorem, we obtain 
which infers that

$$
\left\langle I_{\lambda, \mu}^{\prime}(\zeta(w)(u-w)), \zeta(w)(u-w)\right\rangle=0,
$$

$$
w \in H_{0}^{1}(\Omega),
$$

so $(\zeta(w)(u-w)) \in \mathscr{M}_{\lambda, \mu}$.

\section{Proof of Theorem 1}

According to Lemma 16, we can derive that the problem (1) has a positive solution.

\section{Proof of Theorem 2}

Proposition 18. (i) For every $\lambda \in\left(0, \lambda_{*}\right)$, there exists $(P S)_{\alpha_{\lambda^{*}}}$ sequence $\left\{u_{n}\right\} \subset \mathscr{M}_{\lambda, \mu}$ in $H_{0}^{1}(\Omega)$ for $I_{\lambda, \mu}$;

(ii)For every $\lambda \in\left(0,(q / 2) \lambda_{*}\right)$, there exists $(P S)_{\alpha_{\lambda, \mu}^{-}}$sequence $\left\{u_{n}\right\} \subset \mathscr{M}_{\lambda, \mu}^{-}$in $H_{0}^{1}(\Omega)$ for $I_{\lambda, \mu}$.

Proof. By Lemma 9 and Ekeland variational principle there exists a minimizing sequence $\left\{u_{n}\right\} \subset \mathscr{M}_{\lambda, \mu}$ such that

$$
\begin{aligned}
& I_{\lambda, \mu}\left(u_{n}\right)<\alpha_{\lambda, \mu}+\frac{1}{n}, \\
& I_{\lambda, \mu}\left(u_{n}\right)<\mathrm{I}_{\lambda, \mu}(w)+\frac{1}{n}\left\|w-u_{n}\right\| \quad \forall w \in \mathscr{M}_{\lambda, \mu} .
\end{aligned}
$$

$n$ is large enough, from Theorem 14(i), which yields that

$$
\begin{aligned}
I_{\lambda, \mu}\left(u_{n}\right)= & \left(\frac{1}{2}-\frac{1}{22_{\mu}^{*}}\right)\left\|u_{n}\right\|^{2} \\
& -\left(\frac{1}{q}-\frac{1}{22_{\mu}^{*}}\right) \int_{\Omega} f_{\lambda}|u|^{q}<\alpha_{\lambda, \mu}+\frac{1}{n} \\
< & \frac{q-2}{2 q} \int_{\Omega} f_{\lambda}|u|^{q},
\end{aligned}
$$

which derives that

$$
\begin{aligned}
& \lambda\|f\|_{\infty} S^{-q / 2}|\Omega|^{\left(2^{*}-q\right) / 2^{*}}\left\|u_{n}\right\|^{q} \geq \int_{\Omega} f_{\lambda}\left|u_{n}\right|^{q} \\
& >\frac{2 q}{q-2}\left(\beta_{1}+o(1)\right) .
\end{aligned}
$$

Hence $u \neq \equiv$ by (70). From Lemma 15, we have

$$
\begin{aligned}
\|u\| & >\left[\frac{2 q}{q-2}\left(\beta_{1}+o(1)\right)|\Omega|^{-\left(2_{\mu}^{*}-q\right) / 2_{\mu}^{*}} S^{q / 2}\right]^{1 / q} ; \\
\|u\| & <\left[\frac{q-22_{\mu}^{*}}{2-22_{\mu}^{*}} \lambda|\Omega|^{\left(2^{*}-q\right) / 2^{*}}\left(S_{H, L} C(N, \mu)^{1 / 2_{\mu}^{*}}\right)^{-q / 2}\right. \\
\left.\cdot\left|f_{+}\right|_{\infty}\right]^{1 /(2-q)} &
\end{aligned}
$$

We claim that $\lim _{n \rightarrow \infty}\left\|I_{\lambda, \mu}^{\prime}\left(u_{n}\right)\right\|=0$. Using Lemma 17, then there exists a differentiable function $\zeta_{n}: B\left(0 ; \epsilon_{n}\right) \rightarrow \mathbb{R}^{+}$for some small enough number $\epsilon_{n}>0$, such that $\zeta_{n}(w)\left(u_{n}-w\right) \epsilon$ $\mathscr{M}_{\lambda, \mu}$. Take $\varrho \in\left(0, \epsilon_{n}\right)$, and let $u \in H_{0}^{1}(\Omega)(u \neq 0), w=$ $\varrho\left(I_{\lambda, \mu}^{\prime}\left(u_{n}\right) /\left\|I_{\lambda, \mu}^{\prime}\left(u_{n}\right)\right\|_{H^{-1}}\right), w_{\varrho}=\zeta_{n}(w)\left(u_{n}-w\right)$. Coupling with (68), for $w_{\varrho} \in \mathscr{M}_{\lambda, \mu}$, we obtain

$$
\begin{aligned}
& \frac{1}{n}\left\|w_{\varrho}-u_{n}\right\| \geq I_{\lambda, \mu}\left(u_{n}\right)-I_{\lambda, \mu}\left(w_{\varrho}\right) \\
& =\left(1-\zeta_{n}(\varrho)\right)\left\langle I_{\lambda, \mu}^{\prime}\left(w_{\varrho}\right), u_{n}\right\rangle \\
& \quad+\varrho \zeta_{n}(\varrho)\left\langle I_{\lambda, \mu}^{\prime}\left(w_{\varrho}\right), \frac{I_{\lambda, \mu}^{\prime}\left(u_{n}\right)}{\left\|I_{\lambda, \mu}^{\prime}\left(u_{n}\right)\right\|_{H^{-1}}}+o(\varrho)\right\rangle, \\
& \lim _{\varrho \rightarrow 0} \frac{1}{\varrho}\left\|w_{\varrho}-u_{n}\right\|=\lim _{\varrho \rightarrow 0} \frac{1}{w}\left\|\left(\zeta_{n}(\varrho)-1\right) u_{n}-\zeta_{n}(w) w\right\| \\
& =\lim _{\varrho \rightarrow 0}\left\|\frac{1}{\varrho}\left(\zeta_{n}(w)-1\right) u_{n}-\zeta_{n}(w) \frac{I_{\lambda, \mu}^{\prime}\left(u_{n}\right)}{\left\|I_{\lambda, \mu}^{\prime}\left(u_{n}\right)\right\|_{H^{-1}}}\right\| \\
& \leq\left|\zeta_{n}^{\prime}(0)\right|\left\|u_{n}\right\|+1 .
\end{aligned}
$$

where

$$
\zeta_{n}^{\prime}(0):=\left(\zeta^{\prime}(0) \cdot \frac{I_{\lambda, \mu}^{\prime}}{\left\|I_{\lambda, \mu}^{\prime}\left(u_{n}\right)\right\|_{H^{-1}}}\right) .
$$

Dividing by $\varrho>0$ in (73) and passing to the limit as $\varrho \rightarrow 0$, which infers that

$$
\begin{aligned}
\frac{1}{n}\left(1+\left|\zeta_{n}^{\prime}(0)\right|\left\|u_{n}\right\|\right) \geq & -\zeta_{n}^{\prime}(0)\left\langle I_{\lambda, \mu}^{\prime}\left(u_{n}\right), u_{n}\right\rangle \\
& +\left\|I_{\lambda, \mu}^{\prime}\left(u_{n}\right)\right\|_{H^{-1}} \\
= & \left\|I_{\lambda, \mu}^{\prime}\left(u_{n}\right)\right\|_{H^{-1}} .
\end{aligned}
$$

Applying (71), (72), we have

$$
\left\|I_{\lambda, \mu}^{\prime}\left(u_{n}\right)\right\| \leq \frac{C^{\prime}}{n}\left(1+\left|\zeta_{n}^{\prime}(0)\right|\right) .
$$




\section{Noting that}

$$
\left\langle\zeta_{n}^{\prime}(0), w\right\rangle=\frac{2 \int_{\Omega} \nabla u_{n} \nabla w-22_{\mu}^{*} \int_{\Omega} \int_{\Omega}\left|u_{n}(x)\right|^{2_{\mu}^{*}}\left|u_{n}(y)\right|^{2_{\mu}^{*}-2} u_{n}(y) w(y) /|x-y|^{\mu} d x d y-q \int_{\Omega} f_{\lambda}\left|u_{n}\right|^{q-2} u w}{(2-q)\left\|u_{n}\right\|^{2}-\left(22_{\mu}^{*}-q\right) \int_{\Omega} \int_{\Omega}\left|u_{n}(x)\right|^{2_{\mu}^{*}}\left|u_{n}(y)\right|^{2_{\mu}^{*}} /|x-y|^{\mu} d x d y} .
$$

By Lemma 17, for some constant $C^{\prime \prime}>0$, we have

$$
\left|\zeta_{n}^{\prime}(0)\right| \leq \frac{C^{\prime \prime}\|w\|}{(2-q)\left\|u_{n}\right\|^{2}-\left(22_{\mu}^{*}-q\right) \int_{\Omega} \int_{\Omega}\left(\left|u_{n}(x)\right|^{2_{\mu}^{*}}\left|u_{n}(y)\right|^{2_{\mu}^{*}} /|x-y|^{\mu}\right) d x d y} .
$$

Now we prove that

$$
\begin{aligned}
& (2-q)\left\|u_{n}\right\|^{2} \\
& \quad-\left(22_{\mu}^{*}-q\right) \int_{\Omega} \int_{\Omega} \frac{\left|u_{n}(x)\right|^{2_{\mu}^{*}}\left|u_{n}(y)\right|^{2_{\mu}^{*}}}{|x-y|^{\mu}} d x d y
\end{aligned}
$$

$>c, \quad(c>0)$.

Arguing by contradiction, assume that there exists a subsequence $\left\{u_{n}\right\}$ such that

$$
\begin{aligned}
& \mid(2-q)\left\|u_{n}\right\|^{2} \\
& \quad-\left(22_{\mu}^{*}-q\right) \int_{\Omega} \int_{\Omega} \frac{\left|u_{n}(x)\right|^{2_{\mu}^{*}}\left|u_{n}(y)\right|^{2_{\mu}^{*}}}{|x-y|^{\mu}} d x d y \mid \\
& \quad=o(1) .
\end{aligned}
$$

From Lemma 17 and (72), there exists a constant $v>0$ such that

$$
\begin{aligned}
& \int_{\Omega} \int_{\Omega} \frac{\left|u_{n}(x)\right|^{2_{\mu}^{*}}\left|u_{n}(y)\right|^{2_{\mu}^{*}}}{|x-y|^{\mu}} d x d y \geq v, \\
& \quad \text { for } n \text { sufficiently } \\
& \int_{\Omega} f_{\lambda}\left|u_{n}\right|^{q} \\
& =\left\|u_{n}\right\|^{2}-\int_{\Omega} \int_{\Omega} \frac{\left|u_{n}(x)\right|^{2_{\mu}^{*}}\left|u_{n}(y)\right|^{2_{\mu}^{*}}}{|x-y|^{\mu}} d x d y \\
& =\frac{22_{\mu}^{*}-2}{2-q} \int_{\Omega} \int_{\Omega} \frac{\left|u_{n}(x)\right|^{2_{\mu}^{*}}\left|u_{n}(y)\right|^{2_{\mu}^{*}}}{|x-y|^{\mu}} d x d y \\
& \quad+o(1) .
\end{aligned}
$$$$
\text { for } n \text { sufficiently large, }
$$

\section{Moreover}

$$
\begin{aligned}
\Psi & \left(u_{n}\right) \\
& =\left(\int_{\Omega} \int_{\Omega} \frac{\left|u_{n}(x)\right|^{2_{\mu}^{*}}\left|u_{n}(y)\right|^{2_{\mu}^{*}}}{|x-y|^{\mu}} d x d y\right)^{\left(2_{\mu}^{*}-q+2\right) / 2_{\mu}^{*}\left(22_{\mu}^{*}-2\right)} \\
& \cdot\left(C(N, \mu) \|\left. w_{n}\right|^{2}-\int_{\Omega} f_{\lambda}\left|w_{n}\right|^{q}\right) \\
& \geq \vartheta\left(\int_{\Omega} \int_{\Omega} \frac{\left|u_{n}(x)\right|^{2 *}\left|u_{n}(y)\right|^{2_{\mu}^{*}}}{|x-y|^{\mu}} d x d y\right)^{\left(2_{\mu}^{*}-q+2\right) / 2_{\mu}^{*}\left(22_{\mu}^{*}-2\right)} \\
& \geq \vartheta \vartheta v^{\left(2_{\mu}^{*}-q+2\right) /\left(2_{\mu}^{*}\left(22_{\mu}^{*}-2\right)\right)}>0,
\end{aligned}
$$


On the other hand, combining (82) with (83), we conclude that

$$
\begin{aligned}
& \Phi\left(u_{n}\right)=C(N, \mu)\left\|u_{n}\right\|^{2\left(22_{\mu}^{*}-q\right) /\left(22_{\mu}^{*}-2\right)} \\
& -\frac{22_{\mu}^{*}-2}{2-q}\left(\int_{\Omega} \int_{\Omega} \frac{\left|u_{n}(x)\right|^{2_{\mu}^{*}}\left|u_{n}(y)\right|^{2_{\mu}^{*}}}{|x-y|^{\mu}} d x d y\right)^{\left(22_{\mu}^{*}-q\right) /\left(22_{\mu}^{*}-2\right)} \\
& +o(1)=\left(\frac{2-q}{22_{\mu}^{*}-q}\right)^{\left(22_{\mu}^{*}-q\right) /\left(22_{\mu}^{*}-2\right)} \\
& \cdot \frac{22_{\mu}^{*}-q}{2-q}\left\|u_{n}\right\|^{2\left(22_{\mu}^{*}-q\right) /\left(22_{\mu}^{*}-2\right)} \\
& -\frac{22_{\mu}^{*}-q}{2-q}\left(\int_{\Omega} \int_{\Omega} \frac{\left|u_{n}(x)\right|^{2_{\mu}^{*}}\left|u_{n}(y)\right|^{2_{\mu}^{*}}}{|x-y|^{\mu}} d x d y\right)^{\left(22_{\mu}^{*}-q\right) /\left(22_{\mu}^{*}-2\right)} \\
& +o(1)=\frac{22_{\mu}^{*}-2}{2-q}\left[\left(\frac{2-q}{22_{\mu}^{*}-q}\left\|u_{n}\right\|\right)^{\left(22_{\mu}^{*}-q\right) /\left(22_{\mu}^{*}-2\right)}\right. \\
& \left.-\left(\int_{\Omega} \int_{\Omega} \frac{\left|u_{n}(x)\right|^{2_{\mu}^{*}}\left|u_{n}(y)\right|^{2_{\mu}^{*}}}{|x-y|^{\mu}} d x d y\right)^{\left(22_{\mu}^{*}-q\right) /\left(22_{\mu}^{*}-2\right)}\right] \\
& =o(1) \text {, }
\end{aligned}
$$

which is a contradiction; thus (80) is true.

Using the same methods to prove (ii). The proof is completed.

Lemma 19. Assume that $\left(f_{1}\right)$ and $\left(g_{1}\right)$ hold, if $\left\{u_{n}\right\}$ is a $(P S)_{\rho}$ sequence, for $I_{\lambda, \mu}$ with $u_{n} \rightarrow u$ in $H_{0}^{1}(\Omega)$, then $I_{\lambda, \mu}^{\prime}(u)=0$ and there exists a constant $C_{1}$, such that $I_{\lambda, \mu}(u) \geq-C_{1} \lambda^{2 /(2-q)}$.

Proof. Since $\left\{u_{n}\right\}$ is a $(P S)_{\rho}$ sequence for $I_{\lambda, \mu}$ with $u_{n} \rightarrow u$ in $H_{0}^{1}(\Omega)$. Obviously,

$$
\begin{aligned}
\left\langle I_{\lambda, \mu}^{\prime}(u), u\right\rangle & =0 \\
\int_{\Omega} \int_{\Omega} \frac{|u(x)|^{2_{\mu}^{*}}|u(y)|^{2_{\mu}^{*}}}{|x-y|^{\mu}} d x d y & =\|u\|^{2}-\int_{\Omega} f_{\lambda}|u|^{q} .
\end{aligned}
$$

Thus

$$
I_{\lambda, \mu}=\left(\frac{1}{2}-\frac{1}{22_{\mu}^{*}}\right)\|u\|^{2}-\left(\frac{1}{q}-\frac{1}{22_{\mu}^{*}}\right) \int_{\Omega} f_{\lambda}|u|^{q} .
$$

Therefore

$$
\begin{aligned}
I_{\lambda, \mu}(u)=\left(\frac{1}{2}-\frac{1}{22_{\mu}^{*}}\right)\|u\|^{2}-\left(\frac{1}{q}-\frac{1}{22_{\mu}^{*}}\right) \int_{\Omega} f_{\lambda}|u|^{q} \\
\geq\left(\frac{1}{2}-\frac{1}{22_{\mu}^{*}}\right)\|u\|^{2}-\left(\frac{1}{q}-\frac{1}{22_{\mu}^{*}}\right) \lambda \int_{\Omega} f_{+}|u|^{q} \\
\geq\left(\frac{1}{2}-\frac{1}{22_{\mu}^{*}}\right)\|u\|^{2}-\left(\frac{1}{q}-\frac{1}{22_{\mu}^{*}}\right) \lambda|\Omega|^{\left(2^{*}-q\right) / 2^{*}} \\
\cdot\left\|f_{+}\right\|{ }_{\infty}\left(S_{H, L} C(N, \mu)^{1 / 2_{\mu}^{*}}\right)^{-q / 2}\|u\|^{q} \geq\left(\frac{1}{2}\right. \\
\left.-\frac{1}{22_{\mu}^{*}}\right)\|u\|^{2}-\frac{2}{q}\left(\left[\frac{2\left(2_{\mu}^{*}-1\right)}{22_{\mu}^{*} q}\right]^{q / 2}\|u\|^{q}\right)^{2 / q} \\
\cdot \frac{2-q}{2}\left\{\frac{\left(22_{\mu}^{*}-q\right)\left(22_{\mu}^{*} q\right)^{(q-2) / 2}}{\left[2\left(2_{\mu}^{*}-1\right)\right]^{q / 2}}\left|f_{+}\right|_{\infty}\right. \\
\left.-\mid 2^{*}-q\right) / 2^{*}\left(S_{H, L} C(N, \mu)^{1 / 2_{\mu}^{*}}\right)^{-q / 2} \\
=-C_{1} \lambda^{2 /(2-q)},
\end{aligned}
$$

where $C_{1}=((2-q) / 2)\left\{\left(\left(22_{\mu}^{*}-q\right)\left(22_{\mu}^{*} q\right)^{(q-2) / 2} /\left[2\left(2_{\mu}^{*}-\right.\right.\right.\right.$ $\left.\left.1)]^{q / 2}\right)\left|f_{+}\right|_{\infty}|\Omega|^{\left(2^{*}-q\right) / 2^{*}}\left(S_{H, L} C(N, \mu)^{1 / 2_{\mu}^{*}}\right)^{-q / 2}\right\}^{2 /(2-q)}$.

The proof is completed.

Lemma 20. Assume that $\left(f_{1}\right),\left(g_{1}\right)$ hold. For $c \in\left(-\infty,\left(\left(2_{\mu}^{*}-\right.\right.\right.$ $\left.\left.1) / 22_{\mu}^{*}\right)\left|g_{+}\right|_{\infty}^{-(N-2) /(N-\mu+2)} S_{H, L}^{(2 N-\mu) /(N-\mu+2)}-C_{1} \lambda^{2 /(2-q)}\right), I_{\lambda, \mu}$ satisfies the $(P S)_{c}$ condition.

Proof. Let $\left\{u_{n}\right\} \subset H$ be a $(P S)_{c}$ sequence, which implies

$$
\begin{aligned}
& I_{\lambda, \mu}\left(u_{n}\right)=c+o(1), \\
& I_{\lambda, \mu}^{\prime}\left(u_{n}\right)=o(1) .
\end{aligned}
$$

By Lemma 9, $\left\{u_{n}\right\}$ is bounded in $H_{0}^{1}(\Omega)$, so there exists a subsequence still denoted by $\left\{u_{n}\right\}$, we can suppose that there exists $u \in H_{0}^{1}(\Omega)$ such that

$$
\begin{aligned}
u_{n} & \rightarrow u, \quad \text { in } H_{0}^{1}(\Omega), \\
u_{n} & \longrightarrow u, \quad \text { in } L^{p}(\Omega), 1 \leq p<2^{*}, \\
u_{n}(x) & \longrightarrow u(x), \text { a.e. in } \Omega .
\end{aligned}
$$

By $\left(f_{1}\right),\left(g_{1}\right)$ and Lemma 19, we know that $I_{\lambda, \mu}^{\prime}(u)=0$ and

$$
\int_{\Omega} f_{\lambda}\left|u_{n}\right|^{q}=\int_{\Omega} f_{\lambda}|u|^{q}+o(1)
$$


Let $w_{n}=u_{n}-u$. According to Brézis-Lieb Lemma and the Vitai's theorem, one has

$$
\begin{aligned}
& \left\|w_{n}\right\|^{2}=\left\|u_{n}\right\|^{2}-\|u\|^{2}+o(1) ; \\
& \int_{\Omega} \int_{\Omega} g(x) \frac{\left|w_{n}(x)\right|^{2_{\mu}^{*}}\left|w_{n}(y)\right|^{2_{\mu}^{*}}}{|x-y|^{\mu}} d x d y \\
& \quad \int_{\Omega} \int_{\Omega} g(x) \frac{\left|u_{n}(x)\right|^{2_{\mu}^{*}}\left|u_{n}(y)\right|^{2_{\mu}^{*}}}{|x-y|^{\mu}} d x d y \\
& \quad-\int_{\Omega} \int_{\Omega} g(x) \frac{|u(x)|^{2_{\mu}^{*}}|u(y)|^{2_{\mu}^{*}}}{|x-y|^{\mu}} d x d y+o(1) .
\end{aligned}
$$

Thus

$$
\begin{aligned}
c+ & o(1) \\
= & \frac{1}{2}\left\|w_{n}\right\|^{2} \\
& -\frac{1}{22_{\mu}^{*}} \int_{\Omega} \int_{\Omega} g(x) \frac{\left|w_{n}(x)\right|^{2_{\mu}^{*}}\left|w_{n}(y)\right|^{2_{\mu}^{*}}}{|x-y|^{\mu}} d x d y \\
& +I_{\lambda, \mu}(u), \\
\left\|w_{n}\right\|^{2} & -\int_{\Omega} \int_{\Omega} g(x) \frac{\left|w_{n}(x)\right|^{2_{\mu}^{*}}\left|w_{n}(y)\right|^{2_{\mu}^{*}}}{|x-y|^{\mu}} d x d y \\
= & o(1) .
\end{aligned}
$$

Therefore, we suppose that

$$
\begin{aligned}
& \lim _{n \rightarrow \infty}\left\|w_{n}\right\|^{2} \\
& \quad=\lim _{n \rightarrow \infty} \int_{\Omega} \int_{\Omega} g(x) \frac{\left|w_{n}(x)\right|^{2_{\mu}^{*}}\left|w_{n}(y)\right|^{2_{\mu}^{*}}}{|x-y|^{\mu}} d x d y=l .
\end{aligned}
$$

By the Sobolev inequality, one has

$$
\left\|w_{n}\right\|^{2} \geq S\left\|w_{n}\right\|_{2_{\mu}^{*}}^{2}
$$

Applying (94), we deduce that

$$
l \leq\left|g_{+}\right|_{\infty}\left(l S_{H, L}^{-1}\right)^{2_{\mu}^{*}}
$$

and, obviously, $l=0$, or $l=\left|g_{+}\right|_{\infty}^{-(N-2) /(2+N-\mu)} S_{H, L}^{(2 N-\mu) /(N-\mu+2)}$. In fact, $l=0$. Arguing by contradiction, suppose $l \geq$ $\left|g_{+}\right|_{\infty}^{-(N-2) /(2+N-\mu)} S_{H, L}^{(2 N-\mu) /(N-\mu+2)}$.
Coupling with (91)-(94), we have

$$
\begin{aligned}
c= & I_{\lambda, \mu}\left(w_{n}\right)-\frac{1}{22_{\mu}^{*}}\left\langle I_{\lambda, \mu}^{\prime}\left(w_{n}\right), w_{n}\right\rangle+o(1) \\
= & \left(\frac{1}{2}-\frac{1}{22_{\mu}^{*}}\right)\left\|w_{n}\right\|^{2}-\left(\frac{1}{q}-\frac{1}{22_{\mu}^{*}}\right) \int_{\Omega} f_{\lambda}\left|w_{n}\right|^{q} \\
\geq & \frac{2_{\mu}^{*}-1}{22_{\mu}^{*}}\left|g_{+}\right|_{\infty}^{-(N-2) /(N-\mu+2)} S_{H, L}^{(2 N-\mu) /(N-\mu+2)} \\
& -C_{1} \lambda^{2 /(2-q)},
\end{aligned}
$$

which is a contradiction, that is, $u_{n} \rightarrow u$ in $H_{0}^{1}(\Omega)$.

This completes the proof.

Lemma 21. Assume that $\left(f_{1}\right),\left(f_{2}\right),\left(g_{1}\right),\left(g_{2}\right)$ hold, then there exist $v \in H_{0}^{1}(\Omega)$ and $\lambda^{*}>0$, such that, for $\lambda \in\left(0, \lambda^{*}\right)$,

$$
\begin{aligned}
\sup _{t \geq 0} & I_{\lambda, \mu}(t v) \\
< & \frac{2_{\mu}^{*}-1}{22_{\mu}^{*}}\left|g_{+}\right|_{\infty}^{-(N-2) /(N-\mu+2)} S_{H, L}^{(2 N-\mu) /(N-\mu+2)} \\
& \quad-C_{1} \lambda^{2 /(2-q)} .
\end{aligned}
$$

In particular, for every $\lambda \in\left(0, \lambda^{*}\right)$, we have $\alpha_{\lambda, \mu}^{-}<\left(\left(2_{\mu}^{*}-\right.\right.$ $\left.1) / 22_{\mu}^{*}\right)\left|g_{+}\right|_{\infty}^{-(N-2) /(N-\mu+2)} S_{H, L}^{(2 N-\mu) /(N-\mu+2)}-C_{1} \lambda^{2 /(2-q)}$.

Proof. Set

$H(u)$

$$
=\frac{1}{2}\|u\|^{2}-\frac{1}{22_{\mu}^{*}} \int_{\Omega} \int_{\Omega} g(x) \frac{|u(x)|^{2_{\mu}^{*}}|u(y)|^{2_{\mu}^{*}}}{|x-y|^{\mu}} d x d y,
$$

$K(u)$

$$
=\frac{\|u\|^{2}}{\left(\int_{\Omega} \int_{\Omega} g(x)\left(|u(x)|^{2_{\mu}^{*}}|u(y)|^{2_{\mu}^{*}} /|x-y|^{\mu}\right) d x d y\right)^{1 / 2_{\mu}^{*}} .}
$$

Based on $\left(g_{2}\right)$, we get that there exists $\delta \in\left(0, \rho_{0}\right)$, for every $x \in B(0,2 \delta)$ such that

$$
g(x)-g(0)=o\left(|x|^{\beta}\right) .
$$


For $\varepsilon>0$, let $\eta \in C_{0}^{\infty}(\Omega)$ be a cut function, such that

$$
\begin{aligned}
0 & \leq \eta \leq 1, \\
\eta(x) & =\left\{\begin{array}{ll}
1, & |x| \leq \delta, \\
0, & |x|>2 \delta,
\end{array} \quad|\nabla \eta| \leq C .\right.
\end{aligned}
$$

Let

$$
u_{\varepsilon}(x)=\eta(x) U_{\varepsilon}(x)
$$

where

$$
\begin{aligned}
U_{\varepsilon}(x) & =\varepsilon^{(2-N) / 2} U\left(\frac{x}{\varepsilon}\right), \\
U(x) & =\frac{[N(N-2)]^{(N-2) / 4}}{\left(1+|x|^{2}\right)^{(N-2) / 2}} .
\end{aligned}
$$

Step 1. We will show that $\sup _{t>0} H\left(t u_{\varepsilon}\right) \leq((N-\mu+2) /(2(2 N-$ $\mu)))\left|g_{+}\right|_{\infty}^{-(N-2) /(2+N-\mu)} S_{H, L}^{(2 N-\mu) /(N+2-\mu)}+O\left(\varepsilon^{N-2}\right)$.
At first, we prove the following estimates $(\varepsilon \rightarrow 0)$ :

$$
\begin{aligned}
& \left(\int_{\Omega} \int_{\Omega} g(x) \frac{\left|u_{\varepsilon}(x)\right|^{2_{\mu}^{*}}\left|u_{\varepsilon}(y)\right|^{2_{\mu}^{*}}}{|x-y|^{\mu}} d x d y\right)^{1 / 2_{\mu}^{*}} \\
& \quad \leq \varepsilon^{2-N}\left|g_{+}\right|_{\infty}^{(N-2) /(2 N-\mu)} C(N, \mu)^{(N(N-2)) /(2(2 N-\mu))} \\
& \cdot S_{H, L}^{(N-2) / 2}+O\left(\varepsilon^{N-2}\right) ; \\
& \left\|u_{\varepsilon}\right\|^{2}=C(N, \mu)^{N / 22_{\mu}^{*}} S_{H, L}^{N / 2}+O\left(\varepsilon^{N-2}\right) .
\end{aligned}
$$

From [22] and definition of $u_{\varepsilon}$, let $x / i=u,\left(x_{1}, x_{2}, \ldots, x_{n}\right)=$ $i\left(u_{1}, u_{2} \cdots, u_{n}\right) x_{i}=\varepsilon u_{i}, d x_{i}=\varepsilon d u_{i}$, which derives that

$$
\begin{aligned}
& \left(\int_{\Omega} \int_{\Omega} g(x) \frac{\left|u_{\varepsilon}(x)\right|^{2_{\mu}^{*}}\left|u_{\varepsilon}(y)\right|^{2_{\mu}^{*}}}{|x-y|^{\mu}} d x d y\right)^{1 / 2_{\mu}^{*}} \\
& \leq C(N, \mu)^{N / 2} S^{(2 N-\mu) / 2}\left|g_{+}\right|_{\infty}+O\left(\varepsilon^{2 N-\mu}\right) .
\end{aligned}
$$

Furthermore

$$
\begin{aligned}
& \varepsilon^{\mu-2 N}\left|g_{+}\right|_{\infty} C(N, \mu)^{N / 2} S_{H, L}^{(2 N-\mu) / 2}-\int_{\Omega} \int_{\Omega} g(x) \frac{\left|u_{\varepsilon}(x)\right|^{2_{\mu}^{*}}\left|u_{\varepsilon}(y)\right|^{2_{\mu}^{*}}}{|x-y|^{\mu}} d x d y \\
& =\int_{\mathbb{R}^{N} \backslash B(0, \delta)} \int_{\mathbb{R}^{N} \backslash B(0, \delta)} \frac{g(0)-g(x) \eta^{2_{\mu}^{*}}(x)}{\left(1+|x / \varepsilon|^{2}\right)^{(2 N-\mu) / 2}|x-y|^{\mu}\left(1+|y / \varepsilon|^{2}\right)^{(2 N-\mu) / 2}} \\
& +2 \int_{\mathbb{R}^{N} \backslash B(0, \delta)} \int_{B(0, \delta)} \frac{g(0)-g(x) \eta^{2_{\mu}^{*}}(x)}{\left(\varepsilon^{2}+|x|^{2}\right)^{(2 N-\mu) / 2}|x-y|^{\mu}\left(\varepsilon^{2}+|y|^{2}\right)^{(2 N-\mu) / 2}} \\
& +\int_{B(0, \delta)} \int_{B(0, \delta)} \frac{g(0)-g(x)}{\left(1+|x / \varepsilon|^{2}\right)^{(2 N-\mu) / 2}|x-y|^{\mu}\left(1+|y / \varepsilon|^{2}\right)^{(2 N-\mu) / 2}}-\int_{\Omega} g(x) \frac{\left|u_{\varepsilon}(x)\right|^{2_{\mu}^{*}}\left|u_{\varepsilon}(y)\right|^{2_{\mu}^{*}}}{|x-y|^{\mu}} d x d y=A_{1} \\
& +2 A_{2}+A_{3}-C(N, \mu)^{N / 2} S_{H, L}^{(2 N-\mu) / 2},
\end{aligned}
$$

where

$$
\begin{aligned}
A_{1} & =\int_{\mathbb{R}^{N} \backslash B(0, \delta)} \int_{\mathbb{R}^{N} \backslash B(0, \delta)} \frac{g(0)-g(x) \eta^{2_{\mu}^{*}}(x)}{\left(1+|x / \varepsilon|^{2}\right)^{(2 N-\mu) / 2}|x-y|^{\mu}\left(1+|y / \varepsilon|^{2}\right)^{(2 N-\mu) / 2}} \\
& =\varepsilon^{2 N-\mu}[N(N-2)]^{(2 N-\mu) / 2} \int_{\mathbb{R}^{N} \backslash B(0, \delta)} \int_{\mathbb{R}^{N} \backslash B(0, \delta)} \frac{g(0)}{\left(\varepsilon^{2}+|x|^{2}\right)^{(2 N-\mu) / 2}|x-y|^{\mu}\left(\varepsilon^{2}+|y|^{2}\right)^{(2 N-\mu) / 2}} d x d y \\
& \leq \varepsilon^{2 N-\mu}[N(N-2)]^{(2 N-\mu) / 2} \int_{\mathbb{R}^{\mathbb{N} \backslash B(0, \delta)}} \int_{\mathbb{R}^{\mathbb{N}} \backslash B(0, \delta)} \frac{g(0)}{|x|^{2 N-\mu}|x-y|^{\mu}|y|^{2 N-\mu}} d x d y
\end{aligned}
$$




$$
\begin{aligned}
& \leq \varepsilon^{2 N-\mu}[N(N-2)]^{(2 N-\mu) / 2} \int_{\mathbb{R}^{N} \backslash B(0, \delta)} \frac{g(0)}{|x|^{2 N-\mu}} d x \int_{\mathbb{R}^{N} \mid B(0, \delta)} \frac{g(0)}{|y|^{2 N-\mu}} d y \\
& =N \omega_{N} \varepsilon^{2 N-\mu}[N(N-2)]^{(2 N-\mu) / 2} \int_{\delta}^{\infty} g(0) \frac{r^{N-1}}{r^{2 N-\mu}} d r \int_{\delta}^{\infty} g(0) \frac{r^{N-1}}{r^{2 N-\mu}} d r \\
& =N \omega_{N} \varepsilon^{2 N-\mu}[N(N-2)]^{(2 N-\mu) / 2}\left|g_{+}\right|_{\infty}^{2}\left(\frac{\delta^{\mu-N}}{\mu-N}\right)^{2} ; \\
& A_{2}=\int_{\mathbb{R}^{N} \backslash B(0, \delta)} \int_{B(0, \delta)} \frac{g(0)-g(x) \eta^{2_{\mu}^{*}}(x)}{\left(1+|x / \varepsilon|^{2}\right)^{(2 N-\mu) / 2}|x-y|^{\mu}\left(1+|y / \varepsilon|^{2}\right)^{(2 N-\mu) / 2}} \\
& =\varepsilon^{2 N-\mu}[N(N-2)]^{(2 N-\mu) / 2} \int_{\mathbb{R}^{N} \backslash B(0, \delta)} \int_{B(0, \delta)} \frac{g(0)}{\left(\varepsilon^{2}+|x|^{2}\right)^{(2 N-\mu) / 2}|x-y|^{\mu}\left(\varepsilon^{2}+|y|^{2}\right)^{(2 N-\mu) / 2}} d x d y \\
& \leq \varepsilon^{2 N-\mu}[N(N-2)]^{(2 N-\mu) / 2} \int_{\mathbb{R}^{N} \backslash B(0, \delta)} \int_{\mathbb{R}^{N} \backslash B(0, \delta)} \frac{g(0)}{|x|^{2 N-\mu}|x-y|^{\mu}|y|^{2 N-\mu}} d x d y \\
& \leq \varepsilon^{2 N-\mu}[N(N-2)]^{(2 N-\mu) / 2} \int_{\mathbb{R}^{N} \backslash B(0, \delta)} \frac{g(0)}{|x|^{2 N-\mu}} d x \int_{B(0, \delta)} \frac{g(0)}{|y|^{2 N-\mu}} d y \\
& =N \omega_{N} \varepsilon^{2 N-\mu}[N(N-2)]^{(2 N-\mu) / 2} \int_{\delta}^{\infty} g(0) \frac{r^{N-1}}{r^{2 N-\mu}} d r \int_{0}^{\delta} g(0) \frac{r^{N-1}}{r^{2 N-\mu}} d r \\
& =N \omega_{N} \varepsilon^{2 N-\mu}[N(N-2)]^{(2 N-\mu) / 2}\left|g_{+}\right|_{\infty}^{2}\left(\frac{\delta^{\mu-N}}{\mu-N}\right)^{2} ; \\
& A_{3}=\int_{B(0, \delta)} \int_{B(0, \delta)} \frac{g(0)-g(x)}{\left(1+|x / \varepsilon|^{2}\right)^{(2 N-\mu) / 2}|x-y|^{\mu}\left(1+|y / \varepsilon|^{2}\right)^{(2 N-\mu) / 2}} \\
& =\varepsilon^{2 N-\mu}[N(N-2)]^{(2 N-\mu) / 2} \int_{B(0, \delta)} \int_{B(0, \delta)} \frac{o\left(|x|^{\beta}\right)}{\left(\varepsilon^{2}+|x|^{2}\right)^{(2 N-\mu) / 2}|x-y|^{\mu}\left(\varepsilon^{2}+|y|^{2}\right)^{(2 N-\mu) / 2}} d x d y \\
& \leq \varepsilon^{2 N-\mu}[N(N-2)]^{(2 N-\mu) / 2} \int_{B(0, \delta)} \int_{B(0, \delta)} \frac{o\left(|x|^{\beta}\right)}{|x|^{2 N-\mu}|x-y|^{\mu}|y|^{2 N-\mu}} d x d y \\
& \leq \varepsilon^{2 N-\mu}[N(N-2)]^{(2 N-\mu) / 2} \int_{B(0, \delta)} \frac{o\left(|x|^{\beta}\right)}{|x|^{2 N-\mu}} d x \int_{B(0, \delta)} \frac{o\left(|x|^{\beta}\right)}{|y|^{2 N-\mu}} d y \\
& =N \omega_{N} \varepsilon^{2 N-\mu}[N(N-2)]^{(2 N-\mu) / 2} \int_{\delta}^{\infty} o\left(|r|^{\beta}\right) \frac{r^{N-1}}{r^{2 N-\mu}} d r \int_{\delta}^{\infty} o\left(|r|^{\beta}\right) \frac{r^{N-1}}{r^{2 N-\mu}} d r \\
& =N \omega_{N} \varepsilon^{2 N-\mu}[N(N-2)]^{(2 N-\mu) / 2}\left(\frac{o(1) \delta^{\beta-N+\mu}}{\beta-N+\mu}\right)^{2} .
\end{aligned}
$$

Hence

$$
\begin{array}{ll}
A_{1}+2 A_{2}+A_{3}-C(N, \mu)^{N / 2} S_{H, L}^{(2 N-\mu) / 2}\left|g_{+}\right|_{\infty} & \cdot\left(\frac{\delta^{\mu-N}}{\mu-N}\right)^{2}+N \omega_{N} \varepsilon^{2 N-\mu}[N(N-2)]^{(2 N-\mu) / 2} \\
\leq 3 N \omega_{N} \varepsilon^{2 N-\mu}[N(N-2)]^{(2 N-\mu) / 2}\left|g_{+}\right|_{\infty}^{2} & \cdot\left(\frac{o(1) \delta^{\beta-N+\mu}}{\beta-N+\mu}\right)^{2}-C(N, \mu)^{N / 2} S_{H, L}^{(2 N-\mu) / 2}\left|g_{+}\right|_{\infty} \\
\leq=C_{3} .
\end{array}
$$


$\left(\omega_{N}=2 \pi^{N / 2} / N \Gamma(N / 2)\right.$ is the volume of the unit ball $B(0,1)$ in $\mathbb{R}^{\mathbb{N}}$.)

Thus

$$
\begin{aligned}
0 \leq & \varepsilon^{\mu-2 N}\left|g_{+}\right|_{\infty} C(N, \mu)^{N / 2} S_{H, L}^{(2 N-\mu) / 2} \\
& \quad-\int_{\Omega} \int_{\Omega} g(x) \frac{|u(x)|^{2_{\mu}^{*}}|u(y)|^{2_{\mu}^{*}}}{|x-y|^{\mu}} d x d y \leq C_{3},
\end{aligned}
$$

moreover

$$
\begin{aligned}
0 & \leq 1-\varepsilon^{2 N-\mu}\left|g_{+}\right|_{\infty}^{-1} C(N, \mu)^{-N / 2} \\
& \cdot S_{H, L}^{(\mu-2 N) / 2} \int_{\Omega} \int_{\Omega} g(x) \frac{|u(x)|^{2_{\mu}^{*}}|u(y)|^{2_{\mu}^{*}}}{|x-y|^{\mu}} d x d y \\
& \leq \varepsilon^{2 N-\mu}\left|g_{+}\right|_{\infty}^{-1} C(N, \mu)^{-N / 2} S_{H, L}^{(\mu-2 N) / 2} C_{3},
\end{aligned}
$$

which implies that

$$
\begin{aligned}
1- & \varepsilon^{2 N-\mu}\left|g_{+}\right|_{\infty}^{-1} C(N, \mu)^{-N / 2} S_{H, L}^{(\mu-2 N) / 2} C_{3} \\
& \leq \varepsilon^{2 N-\mu}\left|g_{+}\right|_{\infty}^{-1} C(N, \mu)^{-N / 2} \\
& \cdot S_{H, L}^{-(\mu-2 N) / 2} \int_{\Omega} \int_{\Omega} g(x) \frac{|u(x)|^{2_{\mu}^{*}}|u(y)|^{2_{\mu}^{*}}}{|x-y|^{\mu}} d x d y
\end{aligned}
$$$$
\leq 1 \text {. }
$$

Taking sufficient small $\varepsilon$ such that $\varepsilon^{2 N-\mu}\left|g_{+}\right|_{\infty}^{-1} C(N, \mu)^{-N / 2} S_{H, L}^{(\mu-2 N) / 2} C_{3}<1$, by (114), we have

$$
\begin{gathered}
1-\varepsilon^{2 N-\mu}\left|g_{+}\right|_{\infty}^{-1} C(N, \mu)^{-N / 2} C(N, \mu)^{-N / 2} \\
\cdot S_{H, L}^{(\mu-2 N) / 2} C_{3} \leq\left(1-\varepsilon^{2 N-\mu}\left|g_{+}\right|_{\infty}^{-1} C(N, \mu)^{-N / 2}\right. \\
\left.\cdot C(N, \mu)^{-N / 2} S_{H, L}^{(\mu-2 N) / 2} C_{3}\right)^{1 / 2_{\mu}^{*}} \leq\left(\varepsilon^{2 N-\mu}\left|g_{+}\right|_{\infty}^{-1}\right. \\
\cdot C(N, \mu)^{-N / 2} S_{H, L}^{(\mu-2 N) / 2} \int_{\Omega} \int_{\Omega} g(x)
\end{gathered}
$$

$$
\begin{aligned}
& \left.\cdot \frac{|u(x)|^{2_{\mu}^{*}}|u(y)|^{2_{\mu}^{*}}}{|x-y|^{\mu}} d x d y\right)^{(N-2) /(2 N-\mu)} \\
& =\varepsilon^{N-2}\left|g_{+}\right|_{\infty}^{-(N-2) /(2 N-\mu)} C(N, \mu)^{-N(N-2) / 2(2 N-\mu)} \\
& \cdot S_{H, L}^{-(N-2) / 2}\left(\int_{\Omega} \int_{\Omega} g(x)\right. \\
& \left.\cdot \frac{|u(x)|^{2_{\mu}^{*}}|u(y)|^{2_{\mu}^{*}}}{|x-y|^{\mu}} d x d y\right)^{(N-2) /(2 N-\mu)} \leq 1
\end{aligned}
$$

so

$$
\begin{gathered}
\varepsilon^{2-N}\left|g_{+}\right|_{\infty}^{(N-2) /(2 N-\mu)} C(N, \mu)^{N(N-2) / 2(2 N-\mu)} S_{H, L}^{(N-2) / 2} \\
-\varepsilon^{N-\mu+2}\left|g_{+}\right|_{\infty}^{(-N-2+\mu) /(2 N-\mu)} C(N, \\
\mu)^{N(-N-2+\mu) / 2(2 N-\mu)} S_{H, L}^{(-N-2+\mu) / 2} C_{3} \leq\left(\int_{\Omega} \int_{\Omega} g(x)\right. \\
\left.\cdot \frac{|u(x)|^{2_{\mu}^{*}}|u(y)|^{2_{\mu}^{*}}}{|x-y|^{\mu}} d x d y\right)^{(N-2) /(2 N-\mu)} \\
\leq \varepsilon^{N-2}\left|g_{+}\right|_{\infty}^{(N-2) /(2 N-\mu)} C(N, \mu)^{(N(N-2)) / 2(2 N-\mu)}
\end{gathered}
$$$$
\cdot S_{H, L}^{(N-2) / 2}
$$

that is,

$$
\begin{aligned}
& \left(\int_{\Omega} \int_{\Omega} g(x) \frac{|u(x)|^{2_{\mu}^{*}}|u(y)|^{2_{\mu}^{*}}}{|x-y|^{\mu}} d x d y\right)^{(N-2) /(2 N-\mu)} \\
& =\varepsilon^{2-N}\left|g_{+}\right|_{\infty}^{(N-2) /(2 N-\mu)} C(N, \mu)^{N(N-2) / 2(2 N-\mu)} \\
& \cdot S_{H, L}^{(N-2) / 2}+O\left(\varepsilon^{N+2-\mu}\right) .
\end{aligned}
$$

From (106),(107), we conclude that

$$
\begin{aligned}
K\left(u_{\varepsilon}\right) & =\frac{\left\|u_{\varepsilon}\right\|^{2}}{\left(\int_{\Omega} \int_{\Omega} g(x)\left(|u(x)|^{2_{\mu}^{*}}|u(y)|^{2_{\mu}^{*}} /|x-y|^{\mu}\right) d x d y\right)^{(N-2) /(2 N-\mu)}} \\
& =\frac{C(N, \mu)^{N(N-2) / 2(2 N-\mu)} S_{H, L}^{N / 2}+O\left(\varepsilon^{N-2}\right)}{\varepsilon^{2-N}\left|g_{+}\right|_{\infty}^{(N-2) /(2 N-\mu)} C(N, \mu)^{N(N-2) / 2(2 N-\mu)} S_{H, L}^{(N-2) / 2}+O\left(\varepsilon^{N+2-\mu}\right)} \\
& =\left|g_{+}\right|_{\infty}^{-(N-2) /(2 N-\mu)} \frac{C(N, \mu)^{N(N-2) / 2(2 N-\mu)} S_{H, L}^{N / 2}+O\left(\varepsilon^{N-2}\right)}{\varepsilon^{2-N} C(N, \mu)^{N(N-2) / 2(2 N-\mu)} S_{H, L}^{(N-2) / 2}+O\left(\varepsilon^{N+2-\mu}\right)},
\end{aligned}
$$


moreover

$$
\begin{aligned}
& K\left(u_{\varepsilon}\right)-\left|g_{+}\right|_{\infty}^{-(N-2) /(2 N-\mu)} S_{H, L}=\left|g_{+}\right|_{\infty}^{-(N-2) /(2 N-\mu)}\left[\frac{C(N, \mu)^{N(N-2) / 2(2 N-\mu)} S_{H, L}^{N / 2}+O\left(\varepsilon^{N-2}\right)}{\varepsilon^{2-N} C(N, \mu)^{N(N-2) / 2(2 N-\mu)} S_{H, L}^{(N-2) / 2}+O\left(\varepsilon^{N+2-\mu}\right)}-S_{H, L}\right] \\
& =\left|g_{+}\right|_{\infty}^{-(N-2) /(2 N-\mu)} \frac{C(N, \mu)^{N(N-2) / 2(2 N-\mu)} S_{H, L}^{N / 2}+O\left(\varepsilon^{N-2}\right)-S_{H, L}\left(\varepsilon^{2-N} C(N, \mu)^{N(N-2) / 2(2 N-\mu)} S_{H, L}^{(N-2) / 2}+O\left(\varepsilon^{N+2-\mu}\right)\right)}{\varepsilon^{2-N} C(N, \mu)^{N(N-2) / 2(2 N-\mu)} S_{H, L}^{(N-2) / 2}+O\left(\varepsilon^{N+2-\mu}\right)} \\
& =O\left(\varepsilon^{N-2}\right) .
\end{aligned}
$$

Let

$$
\phi(t)=\frac{t^{2}}{2} a-\frac{t^{22_{\mu}^{*}}}{22_{\mu}^{*}} b, \quad a, b>0,
$$

in fact $\phi^{\prime}(t)=t a-t^{22_{\mu}^{*}-1} b$, and $t=(a / b)^{1 /\left(22_{\mu}^{*}-2\right)}:=t_{\max }$, so

$$
\max _{t \geq 0} \phi(t)=\frac{N+2-\mu}{2(2 N-\mu)}\left(\frac{q}{b^{1 / 2 *}}\right)^{(2 N-\mu) /(N-\mu+2)},
$$

which leads to

$$
\sup _{t \geq 0} H\left(t u_{\varepsilon}\right)=\frac{N-\mu+2}{2(2 N-\mu)} K\left(u_{\varepsilon}\right)^{(2 N-\mu) /(N-\mu+2)} .
$$

From (119), we have

$$
\begin{array}{rl}
\sup _{t \geq 0} & H\left(t u_{\varepsilon}\right) \\
\leq & \frac{N-\mu+2}{2(2 N-\mu)}\left|g_{+}\right|_{\infty}^{-(N-2) /(2+N-\mu)} S_{H, L}^{(2 N-\mu) /(N+2-\mu)} \\
& \quad+O\left(\varepsilon^{N-2}\right) .
\end{array}
$$

Step 2. Let $\varepsilon=\lambda^{2 /(N-2)(2-q)}$, there exists $\lambda_{3}>0$, for all $\lambda \epsilon$ $\left(0, \lambda_{3}\right)$, and we have

$$
\begin{aligned}
\sup _{t \geq 0} & I_{\lambda, \mu}\left(t u_{\varepsilon}\right) \\
< & \frac{2_{\mu}^{*}-1}{22_{\mu}^{*}}\left|g_{+}\right|_{\infty}^{-(N-2) /(N-\mu+2)} S_{H, L}^{(2 N-\mu) /(N-\mu+2)} \\
& -C_{1} \lambda^{2 /(2-q)}:=c_{\lambda} .
\end{aligned}
$$

We denote $\delta_{1}>0$ satisfying

$$
\begin{aligned}
& \frac{2_{\mu}^{*}-1}{22_{\mu}^{*}}\left|g_{+}\right|_{\infty}^{-(N-2) /(N-\mu+2)} S_{H, L}^{(2 N-\mu) /(N-\mu+2)} \\
& -C_{1} \lambda^{2 /(2-q)}>0, \quad \text { for } \lambda \in\left(0, \delta_{1}\right) .
\end{aligned}
$$

By $\left(f_{2}\right),\left(g_{2}\right)$, we obtain that

$$
I_{\lambda, \mu}\left(t u_{\varepsilon}\right) \leq \frac{t^{2}}{2}\left\|u_{\varepsilon}\right\|^{2}, \quad \text { for } t \geq 0, \lambda>0,
$$

which implies that there exists $t_{0} \in(0,1)$ such that

$$
\begin{aligned}
\sup _{0 \leq t \leq t_{0}} & I_{\lambda, \mu}\left(t u_{\varepsilon}\right) \\
\leq & \frac{2_{\mu}^{*}-1}{22_{\mu}^{*}}\left|g_{+}\right|_{\infty}^{-(N-2) /(N-\mu+2)} S_{H, L}^{(2 N-\mu) /(N-\mu+2)} \\
& -C_{1} \lambda^{2 /(2-q)}>0, \quad \text { for } \lambda \in\left(0, \delta_{1}\right) .
\end{aligned}
$$

Moreover, set $0<\varepsilon \leq \delta^{(N-2)(2 q+1) / 2}$, which leads to

$$
\begin{gathered}
\sup _{t \geq t_{0}} I_{\lambda, \mu}\left(t u_{\varepsilon}\right)=\sup _{t \geq t_{0}}\left(H\left(t u_{\varepsilon}\right)-\frac{t^{q}}{q} \int_{\Omega} f_{\lambda}\left|u_{\varepsilon}\right|^{q}\right) \\
\leq \frac{2_{\mu}^{*}-1}{22_{\mu}^{*}}\left|g_{+}\right|_{\infty}^{-(N-2) /(N-\mu+2)} S_{H, L}^{(2 N-\mu) /(N-\mu+2)} \\
+O\left(\varepsilon^{N-2}\right)-\frac{t_{0}^{q}}{q} \beta_{0} \lambda \int_{B(0, \delta)}\left|u_{\varepsilon}\right|^{q} .
\end{gathered}
$$

Furthermore

$$
\begin{aligned}
& \int_{B(0, \delta)}\left|u_{\varepsilon}\right|^{q}=\int_{B(0, \delta)} \varepsilon^{(N-2) / 2}\left|\frac{[N(N-2)]^{(N-2) / 4}}{\left(1+|x / \varepsilon|^{2}\right)^{(N-2) / 2}}\right|^{q} \\
& =\varepsilon^{(N-2) / 2}[N(N-2)]^{(N-2) q / 4} \\
& \cdot \int_{B(0, \delta)}\left|\frac{1}{\left(1+|x / \varepsilon|^{2}\right)^{(N-2) / 2}}\right|^{q} \\
& \leq[N(N-2)]^{q((N-2) / 4)} \delta^{(N-2)(2 q+1) / 2}:=C_{4} .
\end{aligned}
$$

Combining with (128) and (129), for $\varepsilon=\lambda^{2 /(N-2)(2-q)} \epsilon$ $\left(0, \delta^{(N-2)(2 q+1) / 2}\right)$, we have

$$
\begin{aligned}
\sup _{t \geq t_{0}} I_{\lambda, \mu}\left(t u_{\varepsilon}\right) & \\
\leq & \frac{2_{\mu}^{*}-1}{22_{\mu}^{*}}\left|g_{+}\right|_{\infty}^{-(N-2) /(N-\mu+2)} S_{H, L}^{(2 N-\mu) /(N-\mu+2)} \\
& +O\left(\lambda^{2 /(2-q)(N-2)}\right)-\frac{t_{0}^{q}}{q} \beta_{0} C_{4} \lambda .
\end{aligned}
$$


It is easy to find $\delta_{2}>0$ such that

$$
O\left(\lambda^{2 /(2-q)}\right)-\frac{t_{0}^{q}}{q} \beta_{0} C_{4} \lambda<-C_{1} \lambda^{2 /(2-q)},
$$

$$
\text { for } \lambda \in\left(0, \delta_{2}\right) \text {. }
$$

Hence, set $\lambda_{3}=\min \left\{\delta_{1}, \delta^{(N-2)(2 q+1) / 2}, \delta_{2}\right\}>0$ and $\varepsilon=$ $\lambda^{2 /(2-q)(N-2)}$, if $\lambda \in\left(0, \lambda_{3}\right)$, then

$$
\begin{aligned}
\sup _{t \geq 0} & I_{\lambda, \mu}\left(t u_{\varepsilon}\right) \\
< & \frac{2_{\mu}^{*}-1}{22_{\mu}^{*}}\left|g_{+}\right|_{\infty}^{-(N-2) /(N-\mu+2)} S_{H, L}^{(2 N-\mu) /(N-\mu+2)} \\
& -C_{1} \lambda^{2 /(2-q)} .
\end{aligned}
$$

Step 3. For every $\lambda \in\left(0, \lambda_{3}\right)$ then $\alpha_{\lambda, \mu}^{-}<\left(\left(2_{\mu}^{*}-\right.\right.$ 1) $\left./ 22_{\mu}^{*}\right)\left|g_{+}\right|_{\infty}^{-(N-2) /(N-\mu+2)} S_{H, L}^{(2 N-\mu) /(N-\mu+2)}-C_{1} \lambda^{2 /(2-q)}$.

By $\left(f_{2}\right)$ and $\left(g_{2}\right)$, we get

$$
\begin{array}{r}
\int_{\Omega} f_{\lambda}\left|u_{\varepsilon}\right|^{q}>0 \\
\int_{\Omega} \int_{\Omega} g(x) \frac{\left|u_{\varepsilon}(x)\right|^{2_{\mu}^{*}}\left|u_{\varepsilon}(y)\right|^{2_{\mu}^{*}}}{|x-y|^{\mu}}>0 .
\end{array}
$$

Using Lemma 13 and the definition of $\alpha_{\lambda, \mu}^{-}$, we have that for every $\lambda \in\left(0, \lambda_{3}\right)$ there exists $t_{\varepsilon}>0$ such that $t_{\varepsilon} u_{\varepsilon} \in M_{\lambda, \mu}^{-}$and

$$
\begin{aligned}
\alpha_{\lambda, \mu}^{-} \leq & I_{\lambda, \mu}\left(t_{\varepsilon} u_{\varepsilon}\right) \leq \sup _{t \geq 0} I_{\lambda, \mu}\left(t u_{\varepsilon}\right) \\
< & \frac{2_{\mu}^{*}-1}{22_{\mu}^{*}}\left|g_{+}\right|_{\infty}^{-(N-2) /(N-\mu+2)} S_{H, L}^{(2 N-\mu) /(N-\mu+2)} \\
& -C_{1} \lambda^{2 /(2-q)} .
\end{aligned}
$$

The proof of (73) is valid.

Theorem 22. Suppose that $\left(f_{1}\right),\left(f_{2}\right),\left(g_{1}\right),\left(g_{2}\right)$ hold, then there exists $\bar{\lambda}>0\left(\bar{\lambda}=\min \left\{\lambda_{3},(q / 2) \lambda_{*}\right\}\right)$ such that for $\lambda \in(0, \bar{\lambda}), I_{\lambda, \mu}$ has a minimizer $u_{2} \in \mathscr{M}_{\lambda, \mu}^{-}$and

(i) $I_{\lambda, \mu}\left(u_{2}\right)=\alpha_{\lambda, \mu}^{-}$;

(ii) $u_{2}$ is a positive solution of $(1)$ in $H_{0}^{1}(\Omega)$.

Proof. According to Proposition 18 (ii), for any $\lambda \epsilon$ $\left(0,(q / 2) \lambda_{*}\right)$, there exists a $(P S)_{\alpha_{\lambda, \mu}^{-}}$sequence $\left\{u_{n}\right\} \subset \mathscr{M}_{\lambda, \mu}^{-}$ in $H_{0}^{1}(\Omega)$ for $I_{\lambda, \mu}$. In terms of Theorem 14 (ii) and Lemmas 20 and 21, for all $\lambda \in\left(0, \lambda_{3}\right), I_{\lambda, \mu}$ satisfies $\alpha_{\lambda, \mu}^{-}>0$ and $(P S)_{\alpha_{\lambda, \mu}^{-}}$condition. From Lemma $9, I_{\lambda, \mu}$ is coercive on $\mathscr{M}_{\lambda, \mu}$, so $\left\{u_{n}\right\}$ is bounded. Moreover, there exists a subsequence still denoted by $\left\{u_{n}\right\}$, and $u_{2} \in \mathscr{M}_{\lambda, \mu}^{-}$such that $u_{n} \rightarrow u_{2}$ in $H_{0}^{1}(\Omega)$, for $\lambda \in(0, \bar{\lambda}), I_{\lambda, \mu}\left(u_{2}\right)=\alpha_{\lambda, \mu}^{-}$. At last, we apply the same arguments as those of Lemma 16 to prove fore $\lambda \in(0, \bar{\lambda}), u_{2}$ is a positive solution of (1).

The proof is completed.

Proof of Theorem 2. According to Lemma 16 and Theorem 22, we admit that $u_{1} \in \mathscr{M}_{\lambda, \mu}^{+}$and $u_{2} \in \mathscr{M}_{\lambda, \mu}^{-}$are two solutions of (1). It is easy to verify that $u_{1}, u_{2}$ is different because $\mathscr{M}_{\lambda, \mu}^{+} \cup$ $\mathscr{M}_{\lambda, \mu}^{-}=\emptyset$.

The proof is completed.

\section{Proof of Theorem 4}

In this part, we will show the proof of Theorem 4. At first, we consider the following problem:

$$
-\Delta u=\int_{\Omega} \int_{\Omega}\left(\frac{|u(y)|^{2_{\mu}^{*}}}{|x-y|^{2}} d y\right) u^{2_{\mu}^{*}-2} u, \text { in } \Omega,
$$

$$
u \in H_{0}^{1}(\Omega) \text {, }
$$

and the associated energy functional $I^{\infty}$ of $(135)$ in $H_{0}^{1}(\Omega)$ is

$$
\begin{aligned}
I^{\infty}(u) & \\
= & \frac{1}{2}\|u\|^{2} \\
& \quad-\frac{1}{22_{\mu}^{*}} \int_{\Omega} \int_{\Omega} g(x) \frac{|u(x)|^{2_{\mu}^{*}}|u(y)|^{2_{\mu}^{*}}}{|x-y|^{\mu}} d x d y .
\end{aligned}
$$

Recalling that

$$
\begin{aligned}
& \inf _{u \in \mathscr{M}^{\infty}\left(\mathbb{R}^{N}\right)} I^{\infty}(u)=\inf _{u \in M^{\infty}(\Omega)} I^{\infty}(u) \\
& =\frac{N-\mu+2}{2(2 N-\mu)} C(N, \mu)^{N(N-2) / 2(2 N-\mu)} S_{H, L}^{N / 2}, \\
& \quad \Omega \in \mathbb{R}^{\mathbb{N}},
\end{aligned}
$$

where

$$
\begin{aligned}
& \mathscr{M}^{\infty}\left(\mathbb{R}^{N}\right) \\
& \quad=\left\{u \in D^{1.2}\left(\mathbb{R}^{N}\right) \backslash\{0\} \mid\left\langle\left(I^{\infty}\right)^{\prime}(u), u\right\rangle=0\right\} ; \\
& \mathscr{M}^{\infty}(\Omega) \\
& \quad=\left\{u \in H_{0}^{1}(\Omega) \backslash\{0\} \mid\left\langle\left(I^{\infty}\right)^{\prime}(u), u\right\rangle=0\right\} .
\end{aligned}
$$

Lemma 23.

$$
\begin{aligned}
& \inf _{u \in \mathscr{M}_{0, \mu}} I_{0, \mu}(u)=\inf _{u \in \mathscr{M}^{\infty}(\Omega)} I^{\infty}(u) \\
& =\frac{N-\mu+2}{2(2 N-\mu)} C(N, \mu)^{N(N-2) / 2(2 N-\mu)} S_{H, L}^{N / 2} .
\end{aligned}
$$

Moreover, (135) has no positive solution $u_{1}$ satisfying $I_{0, \mu}\left(u_{1}\right)=$ $((N-\mu+2) / 2(2 N-\mu)) C(N, \mu)^{N(N-2) / 2(2 N-\mu)} S_{H, L}^{N / 2}$.

Proof. By Lemma 13, for any $\varepsilon>0$, we can obtain that $t_{0}\left(u_{\varepsilon}\right)>$ $t_{\max }(u)$ and $t_{0}\left(u_{\varepsilon}\right) u_{\varepsilon} \in M_{0, \mu}$ i.e., 


$$
\begin{aligned}
t_{0}\left(u_{\varepsilon}\right)>t_{\max }\left(u_{\varepsilon}\right)=\left(\frac{(2-q)\left\|u_{\varepsilon}\right\|^{2}}{\left(22_{\mu}^{*}-q\right) \int_{\Omega} \int_{\Omega} g(x)\left(\left|t_{0}\left(u_{\varepsilon}\right) u_{\varepsilon}(x)\right|^{2_{\mu}^{*}}\left|t_{0}\left(u_{\varepsilon}\right) u_{\varepsilon}(y)\right|^{2_{\mu}^{*}} /|x-y|^{\mu}\right)^{2}} d x d y\right)^{1 /\left(22_{\mu}^{*}-2\right)} . \\
\left\|t_{0}\left(u_{\varepsilon}\right) u_{\varepsilon}\right\|^{2}=\int_{\Omega} f_{-}\left|t_{0}\left(u_{\varepsilon}\right) u_{\varepsilon}\right|^{q}+\int_{\Omega} \int_{\Omega} g(x) \frac{\left|t_{0}\left(u_{\varepsilon}\right) u_{\varepsilon}(x)\right|^{2_{\mu}^{*}}\left|t_{0}\left(u_{\varepsilon}\right) u_{\varepsilon}(y)\right|^{2_{\mu}^{*}}}{|x-y|^{\mu}} d x d y .
\end{aligned}
$$

Furthermore

$$
\begin{aligned}
& \lim _{\varepsilon \rightarrow 0}\left(\int_{\Omega} \int_{\Omega} g(x) \frac{\left|u_{\varepsilon}(x)\right|^{2_{\mu}^{*}}\left|u_{\varepsilon}(y)\right|^{2_{\mu}^{*}}}{|x-y|^{\mu}} d x d y\right)^{1 / 2_{\mu}^{*}} \\
& \quad=C(N, \mu)^{N(N-2) / 2(2 N-\mu)} S_{H, L}^{(N-2) / 2} \\
& \lim _{\varepsilon \rightarrow 0} \int_{\Omega} f_{-}\left|u_{\varepsilon}\right|^{q}=0 .
\end{aligned}
$$

Using (140)-(143), we have

$$
\lim _{\varepsilon \rightarrow 0} t_{0}\left(u_{\varepsilon}\right)=1
$$

Consequently

$$
\begin{aligned}
& \inf _{u \in \mathscr{M}_{0, \mu}} I_{0, \mu}(u) \leq \lim _{\varepsilon \rightarrow 0} I_{0, \mu}\left(t_{0}\left(u_{\varepsilon}\right) u_{\varepsilon}\right) \\
& =\frac{N-\mu+2}{2(2 N-\mu)} C(N, \mu)^{N(N-2) / 2(2 N-\mu)} S_{H, L}^{N / 2}, \\
& \inf _{u \in \mathscr{M}_{0, \mu}} I_{0, \mu}(u) \leq \inf _{u \in \mathscr{M}^{\infty}(\Omega)} I^{\infty}(u) \\
& =\frac{N-\mu+2}{2(2 N-\mu)} C(N, \mu)^{N(N-2) / 2(2 N-\mu)} S_{H, L}^{N / 2} .
\end{aligned}
$$

Applying Lemma (18)(i), for every $u \in \mathscr{M}_{0, \mu}, I_{0, \mu}(u)=$ $\sup _{t \geq 0} I_{0, \mu}(t u)$. In addition, there exists a unique $t_{3}>0$ such that $t_{3} u \in \mathscr{M}^{\infty}(\Omega)$. Hence

$$
\begin{aligned}
I_{0, \mu}(u) & \geq I_{0, \mu}\left(t_{3} u\right) \geq I^{\infty}\left(t_{3} u\right) \\
& \geq \frac{N-\mu+2}{2(2 N-\mu)} C(N, \mu)^{N(N-2) / 2(2 N-\mu)} S_{H, L}^{N / 2} .
\end{aligned}
$$

Furthermore,

$$
\begin{aligned}
& \inf _{u \in \mathscr{M}_{0, \mu}} I_{0, \mu}(u) \\
& \quad \geq \frac{N-\mu+2}{2(2 N-\mu)} C(N, \mu)^{N(N-2) / 2(2 N-\mu)} S_{H, L}^{N / 2},
\end{aligned}
$$

so

$$
\begin{aligned}
& \inf _{u \in \mathscr{M}_{0, \mu}} I_{0, \mu}(u)=\inf _{u \in \mathscr{M}^{\infty}(\Omega)} I^{\infty}(u) \\
& =\frac{N-\mu+2}{2(2 N-\mu)} C(N, \mu)^{N(N-2) / 2(2 N-\mu)} S_{H, L}^{N / 2}
\end{aligned}
$$

Next, we assume that (135) has a solution $u_{1}$ satisfying $I_{0, \mu}\left(u_{1}\right)=\inf _{u \in \mathscr{M}^{0}} I_{0, \mu}(u)$. By contradiction, suppose there exists $u_{1} \in \mathscr{M}_{0, \mu}$ such that $I_{0, \mu}\left(u_{1}\right)=\inf _{u \in \mathscr{M}_{0, \mu}} I_{0, \mu}(u)$. In fact, $u_{1}$ is a positive solution owing to $I_{0, \mu}\left(u_{1}\right) \stackrel{\mu^{\prime}, \mu}{=} I_{0, \mu}\left(\left|u_{1}\right|\right)$ and $\left|u_{1}\right| \in \mathscr{M}_{0, \mu}$, from Lemmas 10 and 13 , we conclude that $I_{0, \mu}\left(u_{1}\right)=\sup _{t \geq 0} I_{0, \mu}\left(t u_{1}\right)$, and there exists a unique $t_{3}>0$ with $t_{3} u_{1} \in \mathscr{M}^{\infty}(\Omega)$ such that

$$
\begin{aligned}
& \frac{N-\mu+2}{2(2 N-\mu)} C(N, \mu)^{N(N-2) / 2(2 N-\mu)} S_{H, L}^{N / 2} \\
& \quad \inf _{u \in \mathscr{M}_{0, \mu}} I_{0, \mu}(u)=I_{0, \mu}\left(u_{1}\right) \geq I_{0, \mu}\left(t_{3} u_{1}\right) \\
& \geq I^{\infty}\left(t_{3} u_{1}\right)+\frac{t^{22_{\mu}^{*}}}{22_{\mu}^{*}} \\
& \cdot \int_{\Omega} \int_{\Omega}(1-g(x)) \frac{\left|u_{1}(x)\right|^{2_{\mu}^{*}}\left|u_{1}(y)\right|^{2_{\mu}^{*}}}{|x-y|^{\mu}} d x d y \\
& >\frac{N-\mu+2}{2(2 N-\mu)} C(N, \mu)^{N(N-2) / 2(2 N-\mu)} S_{H, L}^{N / 2}+\frac{t^{22_{\mu}^{*}}}{22_{\mu}^{*}} \\
& \cdot \int_{\Omega} \int_{\Omega}(1-g(x)) \frac{\left|u_{1}(x)\right|^{2_{\mu}^{*}}\left|u_{1}(y)\right|^{2_{\mu}^{*}}}{|x-y|^{\mu}} d x d y,
\end{aligned}
$$

which indicates that $\int_{\Omega} \int_{\Omega}(1-g(x))\left(\left|u_{1}(x)\right|^{2_{\mu}^{*}}\left|u_{1}(y)\right|^{2_{\mu}^{*}} / \mid x-\right.$ $\left.\left.y\right|^{\mu}\right) d x d y<0$. That is $1-g<0$, which contradicts with $g \leq 1$. This proof is completed.

Lemma 24. Assume that $\left\{u_{n}\right\}$ is a minimizing sequence for $I_{0, \mu}$ in $\mathscr{M}_{0, \mu}$, then (i) $\int_{\Omega} f_{-}\left|u_{n}\right|^{q}=o(1)$. $o(1)$.

(ii) $\int_{\Omega} \int_{\Omega}(1-g(x))\left(\left|u_{n}(x)\right|^{2_{\mu}^{*}}\left|u_{n}(y)\right|^{2_{\mu}^{*}} /|x-y|^{\mu}\right) d x d y=$ In addition, for $I_{0, \mu}, \quad\left\{u_{n}\right\}$ is a

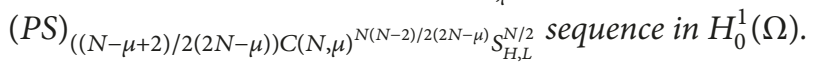

Proof. For any $n$, there exists $t_{n}>0$ such that $t_{n} u_{n} \in \mathscr{M}^{\infty}(\Omega)$, which implies that

$$
t_{n}^{2}\left\|u_{n}\right\|^{2}=t_{n}^{2_{\mu}^{*}} \int_{\Omega}\left|u_{n}\right|^{2_{\mu}^{*}}
$$


Form Lemma 13(i)

$$
\begin{gathered}
I_{0, \mu}\left(u_{n}\right) \geq I_{0, \mu}\left(t_{n} u_{n}\right) \geq I^{\infty}\left(t_{n} u_{n}\right)-\frac{t_{n}^{q}}{q} \int_{\Omega} f_{-}\left|u_{n}\right|^{q} \\
+\frac{t^{22_{\mu}^{*}}}{22_{\mu}^{*}} \int_{\Omega} \int_{\Omega}(1-g(x)) \frac{\left|u_{n}(x)\right|^{2_{\mu}^{*}}\left|u_{n}(y)\right|^{2_{\mu}^{*}}}{|x-y|^{\mu}} d x d y \\
\geq \frac{N-\mu+2}{2(2 N-\mu)} C(N, \mu)^{N(N-2) / 2(2 N-\mu)} S_{H, L}^{N / 2}-\frac{t_{n}^{q}}{q} \\
\cdot \int_{\Omega} f_{-}\left|u_{n}\right|^{q}+\frac{t^{22_{\mu}^{*}}}{22_{\mu}^{*}} \\
\cdot \int_{\Omega} \int_{\Omega}(1-g(x)) \frac{\left|u_{n}(x)\right|^{2_{\mu}^{*}}\left|u_{n}(y)\right|^{2_{\mu}^{*}}}{|x-y|^{\mu}} d x d y .
\end{gathered}
$$

By Lemma 23, we can get

$$
\begin{aligned}
& \frac{t_{n}^{q}}{q} \int_{\Omega} f_{-}\left|u_{n}\right|^{q}=o(1) \\
& \frac{t^{22_{\mu}^{*}}}{22_{\mu}^{*}} \int_{\Omega} \int_{\Omega}(1-g(x)) \frac{\left|u_{n}(x)\right|^{2_{\mu}^{*}}\left|u_{n}(y)\right|^{2_{\mu}^{*}}}{|x-y|^{\mu}} d x d y \\
& \quad=o(1) .
\end{aligned}
$$

Indeed, $\forall n$, there is $C_{5}>0$ such that $t_{n}>C_{5}$. Arguing with contradiction, assume that $t_{n} \rightarrow 0$ as $n \rightarrow \infty$. Noticing that

$$
\begin{aligned}
I_{0, \mu}\left(u_{n}\right)= & \frac{N-\mu+2}{2(2 N-\mu)} C(N, \mu)^{N(N-2) / 2(2 N-\mu)} S_{H, L}^{N / 2} \\
& +o(1), \\
I_{0, \mu}\left(u_{n}\right)= & \frac{N-\mu+2}{2(2 N-\mu)}\left\|u_{n}\right\|^{2}+o(1) .
\end{aligned}
$$

By Lemma 9, we know that $\left\|u_{n}\right\|$ is bounded, thus $\left\|t_{n} u_{n}\right\| \rightarrow 0$ or $I^{\infty}\left(t_{n} u_{n}\right) \rightarrow 0$ which is a contradiction with $I^{\infty}\left(t_{n} u_{n}\right) \geq$ $((N-\mu+2) / 2(2 N-\mu)) C(N, \mu)^{N / 2} S_{H, L}^{(2 N-\mu) / 2}>0$, which derives

$$
\begin{aligned}
& \int_{\Omega} f_{-}\left|u_{n}\right|^{q}=o(1) \\
& \int_{\Omega} \int_{\Omega}(1-g(x)) \frac{\left|u_{n}(x)\right|^{2_{\mu}^{*}}\left|u_{n}(y)\right|^{2_{\mu}^{*}}}{|x-y|^{\mu}} d x d d y \\
& \quad=o(1)
\end{aligned}
$$

and so

$$
\begin{aligned}
& \left\|u_{n}\right\|^{2} \int_{\Omega}\left|u_{n}\right|^{2_{\mu}^{*}}+o(1), \\
& I^{\infty}\left(u_{n}\right)=\frac{N-\mu+2}{2(2 N-\mu)} C(N, \mu)^{N(N-2) / 2(2 N-\mu)} S_{H, L}^{N / 2} \\
& \quad+o(1) .
\end{aligned}
$$

Using the same method as those of (i), we can prove (ii).
Define

$$
\begin{aligned}
& M_{0, \mu}(k)=\left\{u \in \mathscr{M}_{0, \mu} \mid I_{0, \mu}(u)\right. \\
& \quad \leq \frac{N-\mu+2}{2(2 N-\mu)} C(N, \mu)^{N(N-2) / 2(2 N-\mu)} S_{H, L}^{N / 2}+k, k \\
& \quad>0 .\}
\end{aligned}
$$

Let $\Psi: H_{0}^{1}(\Omega) \rightarrow \mathbb{R}^{N}$ be a barycentre map:

$$
\Psi(u)=\frac{\int_{\Omega} x|u|^{2_{\mu}^{*}}}{\int_{\Omega}|u|^{2_{\mu}^{*}}} .
$$

Lemma 25. For $\delta \in\left(0, r_{1}\right)$, there exists $k_{\delta}>0$ such that

$$
\Psi(u) \in G_{\delta}, \quad \forall u \in M_{0, \mu}\left(k_{\delta}\right) .
$$

Proof. According to Lemma 24, there exist a $(P S)_{((N-\mu+2) / 2(2 N-\mu)) C(N, \mu)^{N(N-2) / 2(2 N-\mu)} S_{H, L}^{N / 2}}$ sequence $\left\{u_{n}\right\} \subset M_{0, \mu}$ in $H_{0}^{1}(\Omega)$ and $\delta_{0}<r_{1}$. Since $\Omega$ is bounded and using concentration compactness principle, there exist two sequences $\left\{x_{n}\right\} \subset \Omega$ and $\left\{R_{n}\right\} \subset \mathbb{R}^{+}$with $x_{1} \in \bar{\Omega}$, and $v_{1} \in D^{1,2}\left(\mathbb{R}^{N}\right)$ is a positive solution of (16), such that $x_{n} \rightarrow x_{1}$ and $R_{n} \rightarrow \infty$ as $n \rightarrow \infty$ satisfying

$$
\begin{aligned}
\left\|u_{n}(x)-R_{n}^{(N-2 \mu) / 2} v_{1}\left(R_{n}\left(x-x_{n}\right)\right)\right\|_{D^{1,2}\left(\mathbb{R}^{N}\right)} & \longrightarrow 0, \\
\text { as } n & \longrightarrow \infty .
\end{aligned}
$$

Thus

$$
\begin{aligned}
\Psi\left(u_{n}\right) & =\frac{\int_{\Omega} x\left|u_{n}\right|^{2_{\mu}^{*}}}{\int_{\Omega}\left|u_{n}\right|^{2_{\mu}^{*}}} d x d y \\
& =\frac{\int_{\Omega} x\left|R_{n}^{(N-2 \mu) / 2} v_{1}\left(R_{n}\left(x-x_{n}\right)\right)\right|^{2_{\mu}^{*}}}{\int_{\Omega}\left|R_{n}^{(N-2 \mu) / 2} v_{1}\left(R_{n}\left(x-x_{n}\right)\right)\right|^{2_{\mu}^{*}}}+o(1) \\
& =\frac{\int_{\Omega}\left(\left(x / R_{n}\right)+x_{n}\right)\left|v_{1}(x)\right|^{2_{\mu}^{*}}}{\int_{\Omega}\left|v_{1}(x)\right|^{2_{\mu}^{*}}}+o(1) \\
& =x_{1}+o(1) .
\end{aligned}
$$

In fact, $x_{1} \in G_{\delta_{0}}$. We know that $\left\{u_{n}\right\}$ is a minimizing sequence for $M_{0, \mu}$, and, combining with Lemma 24 (ii), we have

$$
\begin{aligned}
0 & =\lim _{n \rightarrow \infty} \int_{\Omega}(1-g)\left|u_{n}\right|^{2_{\mu}^{*}} \\
& =\lim _{n \rightarrow \infty} \int_{\Omega}(1-g)\left|R_{n}^{(N-2 \mu) / 2} v_{1}\left(R_{n}\left(x-x_{n}\right)\right)\right|^{(N-2 \mu) / 2} \\
& =\lim _{n \rightarrow \infty} \int_{\Omega}(1-g)\left(\frac{x}{R_{n}}+x_{n}\right)\left|v_{1}(x)\right|^{(N-2 \mu) / 2} \\
& =\left(1-g\left(x_{1}\right)\right) S^{N / 2 \mu} \\
& =\left(1-g\left(x_{1}\right)\right)\left(S_{H, L} C(N, \mu)^{1 / 2_{\mu}^{*}}\right)^{N / 2 \mu}
\end{aligned}
$$


which deduce that $g\left(x_{1}\right)=\max _{x \in \bar{\Omega}} g(x)=1$ and

$$
x_{1} \in\left\{x \in \bar{\Omega} \mid g(x)=\max _{y \in \bar{\Omega}} g(y)=1\right\}=G,
$$

and this contradicts with assumption. This completes the proof.

Set

$$
\mathscr{M}_{\lambda, \mu}(c)=\left\{u \in \mathscr{M}_{\lambda, \mu}^{-} \mid I_{\lambda, \mu}(u) \leq c\right\} .
$$

Lemma 26. For every $0<\delta<r_{1}$, there exists $\lambda_{\delta} \in$ $\left(0,(q / 2) \lambda_{*}\right)$, such that, for every $\lambda<\lambda_{\delta}$, we have

$$
\Psi(u) \in G_{\delta}, \quad \text { for every } u \in \mathscr{M}_{\lambda, \mu}\left(c_{\lambda}\right) .
$$

Proof. For $u \in \mathscr{M}_{\lambda, \mu}\left(c_{\lambda}\right)$, combining (26) with Lemma 13(i), there exists a unique $t_{3}>t_{\max }(u)$, such that $t_{3} u \in \mathscr{M}_{0, \mu}$. Based on Sobolev and Höder inequalities, one has

$$
\begin{aligned}
I_{\lambda, \mu}(u)=\sup _{t \geq t_{\max }(u)} I_{\lambda, \mu}(t u) \geq I_{\lambda, \mu}\left(t_{3} u\right) \\
=I_{0, \mu}\left(t_{3} u\right)-\lambda \frac{t_{3}^{q}}{q} \int_{\Omega} f_{+}|u|^{q} \\
\geq I_{0, \mu}\left(t_{3} u\right)-\lambda \frac{t^{q}}{q}\left\|f_{+}\right\|_{L^{q^{*}}} S^{-q / 2}\|u\|^{q} \\
=I_{0, \mu}\left(t_{3} u\right) \\
\quad-\lambda \frac{t_{3}^{q}}{q}\left\|f_{+}\right\|_{\infty}\left(S_{H, L} C(N, \mu)^{1 / 2_{\mu}^{*}}\right)^{-q / 2}\|u\|^{q} .
\end{aligned}
$$

We assume that there exists a positive constant $m$ such that $t_{3} \leq C_{6}$. Using (26) and Sobolev inequality, we deduce that

$$
\begin{aligned}
\|u\|^{2} & \leq \frac{22_{\mu}^{*}-q}{2-q} \int_{\Omega} \int_{\Omega} g(x) \frac{|u(x)|^{2_{\mu}^{*}}|u(y)|^{2_{\mu}^{*}}}{|x-y|^{\mu}} \\
& \leq \frac{22_{\mu}^{*}-q}{2-q} C(N, \mu)^{2_{\mu}^{*}-1}|u|^{22_{\mu}^{*}} \\
& =\frac{22_{\mu}^{*}-q}{2-q} C(N, \mu)^{2_{\mu}^{*}-1}\|u\|^{22_{\mu}^{*}} S_{H, L}^{-2_{\mu}^{*}} .
\end{aligned}
$$

So

$$
\|u\| \geq \frac{(2-q) S_{H, L}^{2_{\mu}^{*}}}{\left(22_{\mu}^{*}-q\right) C(N, \mu)^{2_{\mu}^{*}-1}} .
$$

We may assume that $t_{3} \geq 1$, which leads to

$$
\begin{aligned}
& t_{3}^{22_{\mu}^{*}} \int_{\Omega} \int_{\Omega} g(x) \frac{|u(x)|^{2_{\mu}^{*}}|u(y)|^{2_{\mu}^{*}}}{|x-y|^{\mu}} \\
& \quad=t_{3}^{2}\|u\|^{2}-t_{3}^{q} \int_{\Omega} f_{-}|u|^{q} \\
& \quad \leq t_{3}^{2}\left(\|u\|^{2}+\int_{\Omega}\left|f_{-}\right||u|^{q}\right) .
\end{aligned}
$$

That is

$$
\leq\left(\frac{\|u\|^{2}+\int_{\Omega}\left|f_{-}\right||u|^{q}}{\int_{\Omega} \int_{\Omega} g(x)\left(|u(x)|^{2_{\mu}^{*}}|u(y)|^{2_{\mu}^{*}} /|x-y|^{\mu}\right)}\right)^{1 /\left(22_{\mu}^{*}-2\right)} .
$$

Combining (167), (169), one gets

$$
\begin{aligned}
t_{3} & \leq\left[\frac{22_{\mu}^{*}-q}{2-q}\left(\frac{\|u\|^{2}+\int_{\Omega}\left|f_{-}\right||u|^{q}}{\|u\|^{2}}\right)\right]^{1 /\left(22_{\mu}^{*}-2\right)} \leq\left[\frac{22_{\mu}^{*}-q}{2-q}(1\right. \\
& \left.\left.+\frac{\int_{\Omega}\left|f_{-}\right||u|^{q}}{S^{q / 2}\|u\|^{2-q}}\right)\right]^{1 /\left(22_{\mu}^{*}-2\right)} \leq\left[\frac{22_{\mu}^{*}-q}{2-q}(1\right. \\
& \left.\left.+\frac{\left\|f_{-}\right\|_{\infty}}{\left(S_{H, L} C(N, \mu)^{1 / 2_{\mu}^{*}}\right)^{q / 2}}\|u\|^{2-q}\right)\right]^{1 /\left(22_{\mu}^{*}-2\right)} \leq\left\{\frac{22_{\mu}^{*}-q}{2-q}(1\right. \\
& \left.\left.+\frac{\left\|f_{-}\right\|_{\infty}}{\left(S_{H, L} C(N, \mu)^{1 / 2_{\mu}^{*}}\right)}\left[\frac{(2-q) S_{H, L}^{2_{\mu}^{*}}}{\left(22_{\mu}^{*}-q\right) C(N, \mu)^{2^{*}-1}}\right]\right)\right\}^{1 /\left(22_{\mu}^{*}-2\right)} \\
& :=m .
\end{aligned}
$$

From (165) and (170),

$$
\begin{aligned}
I_{0, \mu}( & \left.t_{3} u\right) \\
\leq & I_{\lambda, \mu}(u) \\
& +\frac{\lambda}{q} t_{3}^{q}\left\|f_{+}\right\|_{\infty}\left(S_{H, L} C(N, \mu)^{1 / 2_{\mu}^{*}}\right)^{-q / 2}\|u\|^{q} \\
\leq & c_{\lambda}+\frac{\lambda m^{q}}{q}\left\|f_{+}\right\|_{\infty}\left(S_{H, L} C(N, \mu)^{1 / 2_{\mu}^{*}}\right)^{-q / 2}\|u\|^{q} .
\end{aligned}
$$

Using Lemma 9, if $I_{\lambda, \mu}(u) \leq c_{\lambda}<((N-\mu+2) / 2(2 N-$ $\mu)) C(N, \mu)^{N(N-2) / 2(2 N-\mu)} S_{H, L}^{N / 2}$. For every $\lambda \in\left(0,(q / 2) \lambda_{*}\right)$, there exists $C_{6}>0$ such that $\|u\| \leq C_{6}$ for every $u \in \mathscr{M}_{\lambda, \mu}\left(c_{\lambda}\right)$. Thus

$$
\begin{aligned}
& I_{0, \mu}\left(t_{3} u\right) \\
& \quad \leq c_{\lambda}+\frac{\lambda m^{q}}{q}\left\|f_{+}\right\|_{\infty}\left(S_{H, L} C(N, \mu)^{1 / 2_{\mu}^{*}}\right)^{-q / 2}\|u\|^{q} .
\end{aligned}
$$

Taking $k_{\delta}>0$, for $\lambda<\lambda_{\delta}$, there exists $\lambda_{\delta} \in\left(0,(q / 2) \lambda_{*}\right)$, and we have

$$
\begin{aligned}
I_{\lambda, \mu}(u) \leq & \frac{N-\mu+2}{2(2 N-\mu)} C(N, \mu)^{(N(N-2)) /(2(2 N-\mu))} S_{H, L}^{N / 2} \\
& +k_{\delta}, \quad \forall u \in \mathscr{M}_{\lambda, \mu}\left(c_{\lambda}\right) .
\end{aligned}
$$

Based on Lemma 25, we derive that $t_{3} u \in \mathscr{M}_{0, \mu}\left(k_{\delta}\right)$ and

$$
\Psi(u)=\frac{\int_{\Omega} x\left|t_{3} u\right|^{2_{\mu}^{*}}}{\int_{\Omega}\left|t_{3} u\right|^{2_{\mu}^{*}}}=\Psi\left(t_{3} u\right) \in G_{\delta},
$$

$\forall u \in M_{\lambda, \mu}\left(c_{\lambda}\right)$. 


$$
\begin{aligned}
& \text { Let } \Gamma(z)=u_{1}+t_{3} u_{\varepsilon} \in \mathscr{M}_{\lambda, \mu}^{-} \text {, and } \\
& \qquad \begin{aligned}
& I_{\lambda, \mu}\left(u_{1}+t_{3} u_{\varepsilon}\right)<c_{\lambda} \\
&= \alpha_{\lambda, \mu}^{+} \\
&+\frac{N-\mu+2}{2(2 N-\mu)} C(N, \mu)^{(N(N-2)) /(2(2 N-\mu))} S_{H, L}^{N / 2} \\
&+k_{\delta},
\end{aligned}
\end{aligned}
$$

so, $u_{1}+t_{3} u_{\varepsilon} \in \mathscr{M}_{\lambda, \mu}\left(c_{\lambda}\right)$. At the same time, we conclude that

$$
\Psi\left(u_{1}+t_{3} u_{\varepsilon}\right) \in G_{\delta} .
$$

Lemma 27 (see [41]). Let closed sets $X, Y, Z$ with $Y \subset Z$ and $h_{1} \subset C(X, Z), h_{2} \subset C(Y, Z)$, and suppose $h_{1} \circ h_{2}$ is homotopically equivalent to identity mapping id in $Z$, then $\operatorname{cat}_{x}(X) \geq \operatorname{cat}_{Z}(Y)$.

Theorem 28. For every $\lambda \in\left(0, \lambda_{\delta}\right), I_{\lambda, \mu}$ has at least cat $G_{\delta}(G)$ critical points on $\mathscr{M}_{\lambda, \mu,+}\left(c_{\lambda}\right)=\left\{u \in I_{\lambda, \mu}\left(c_{\lambda}\right) \mid u \geq 0\right\}$.

Proof. By Lemma 15, for every $\lambda \in\left(0, \lambda_{\delta}\right)$ and $z \in G$

$$
\begin{gathered}
I_{\lambda, \mu}(\Gamma(z))<c_{\lambda} \\
=\alpha_{\lambda, \mu}^{+} \\
\quad+\frac{N-\mu+2}{2(2 N-\mu)} C(N, \mu)^{(N(N-2)) /(2(2 N-\mu))} S_{H, L}^{N / 2}, \\
\Psi(\Gamma(z))=\frac{\int_{\Omega} x|\Gamma(z)|^{2_{\mu}^{*}}}{\int_{\Omega}|\Gamma(z)|^{2_{\mu}^{*}}}=\frac{\int_{\Omega} x\left|u_{2}+t_{z} u_{\varepsilon}\right|^{2_{\mu}^{*}}}{\int_{\Omega}\left|u_{2}+t_{z} u_{\varepsilon}\right|^{2_{\mu}^{*}}} \\
=\frac{\int_{\Omega}(x+z)\left|u_{2}(x+z)+t_{z} \eta(x)\right|^{2_{\mu}^{*}}}{\int_{\Omega}\left|u_{2}(x+z)+t_{z} \eta(x)\right|^{2_{\mu}^{*}}} \\
=z+\frac{\int_{\Omega} z\left|u_{2}(x+z)+t_{\eta}(x) v(x)\right|^{2_{\mu}^{*}}}{\int_{\Omega}\left|u_{2}(x+z)+t_{z} \eta(x) v(x)\right|^{2_{\mu}^{*}}}=z+o(1) \\
\quad(z \longrightarrow 0) .
\end{gathered}
$$

From Lemma 27, $X=\mathscr{M}_{\lambda, \mu}\left(c_{\lambda}\right), Y=G, Z=G_{\delta}, h_{1}=\Psi, h_{2}=$ $\Gamma$, which implies that $\operatorname{cat}_{\mathscr{M}_{\lambda, \mu}}\left(c_{\lambda}\right)\left(\mathscr{M}_{\lambda, \mu}\left(c_{\lambda}\right)>\right.$ cat $\left._{G_{\delta}}(G)\right)$. Combined with Lemma 16, (1) has at least $c a_{G_{\delta}}(G)+1$ solution.

This proof is completed.

\section{Data Availability}

No data were used to support this study.

\section{Conflicts of Interest}

The authors declare that they have no conflicts of interest.

\section{Authors' Contributions}

All authors contributed equally to this work. All authors read and approved the final manuscript.

\section{Acknowledgments}

This project is supported by the Natural Science Foundation of Shanxi Province of China (201601D011003) and the Natural Science Foundation of Shandong Province of China (ZR2017MA036).

\section{References}

[1] C. O. Alves, G. M. Figueiredo, and M. Yang, "Existence of solutions for a nonlinear Choquard equation with potential vanishing at infinity," Advances in Nonlinear Analysis, vol. 5, no. 4, pp. 331-345, 2016.

[2] C. O. Alves, F. Gao, M. Squassina, and M. Yang, "Singularly perturbed critical Choquard equations," Journal of Differential Equations, vol. 263, no. 7, pp. 3943-3988, 2017.

[3] C. O. Alves and M. Yang, "Investigating the multiplicity and concentration behaviour of solutions for a quasi-linear Choquard equation via the penalization method," Proceedings of the Royal Society of Edinburgh, Section: A Mathematics, vol. 146, no. 1, pp. 23-58, 2016.

[4] A. Mao, L. Yang, A. Qian, and S. Luan, "Existence and concentration of solutions of Schrödinger-Possion system," Applied Mathematics Letters, vol. 68, pp. 8-12, 2017.

[5] X. Wang, X. Sun, and W. Lv, "Orbital stability of generalized Choquard equation," Boundary Value Problems, Paper No. 190, 8 pages, 2016.

[6] T. Wang and T. Yi, "Uniqueness of positive solutions of the Choquard type equations," Applicable Analysis: An International Journal, vol. 96, no. 3, pp. 409-417, 2017.

[7] X. Zhang, L. Liu, Y. Wu, and L. Caccetta, "Entire large solutions for a class of Schrodinger systems with a nonlinear random operator," Journal of Mathematical Analysis and Applications, vol. 423, no. 2, pp. 1650-1659, 2015.

[8] A. Mao, R. Jing, S. Luan, J. Chu, and Y. Kong, "Some nonlocal elliptic problem involving positive parameter," Topological Methods in Nonlinear Analysis, vol. 42, no. 1, pp. 207-220, 2013.

[9] X. Zhang, L. Liu, and Y. Wu, "Variational structure and multiple solutions for a fractional advection-dispersion equation," Computers \& Mathematics with Applications. An International Journal, vol. 68, no. 12, part A, pp. 1794-1805, 2014.

[10] K. Li, "Existence of non-trivial solutions for nonlinear fractional Schrödinger-Poisson equations," Applied Mathematics Letters, vol. 72, pp. 1-9, 2017.

[11] A. Qian, "Sing-changing solutions for nonlinear problems with strong resonance," Electronic Journal of Differential Equations, vol. 2012, no. 17, pp. 1-8, 2012.

[12] P. Drabek, A. Kufner, and F. Nicolosi, Quasilinear Elliptic Equations with Degenerations and Singularities, Walter de Gruyter, New York, NY, USA, 1997.

[13] A. Mao, Y. Zhu, and S. Luan, "Existence of solutions of elliptic boundary value problems with mixed type nonlinearities," Boundary Value Problems, 2012:97, 11 pages, 2012.

[14] A. Qian, "Sign-changing solutions for some nonlinear problems with strong resonance," Boundary Value Problems, vol. 2011, pp. 18-1, 2011. 
[15] M. Clapp and B. B. Manna, "Double- and single-layered signchanging solutions to a singularly perturbed elliptic equation concentrating at a single sphere," Communications in Partial Differential Equations, vol. 42, no. 3, pp. 474-490, 2017.

[16] H. Chen and F. Zhou, "Classification of isolated singularities of positive solutions for Choquard equations," Journal of Differential Equations, vol. 261, no. 12, pp. 6668-6698, 2016.

[17] X. Zhang and L. Liu, "The existence and nonexistence of entire positive solutions of semilinear elliptic systems with gradient term," Journal of Mathematical Analysis and Applications, vol. 371, no. 1, pp. 300-308, 2010.

[18] L. Battaglia and J. Van Schaftingen, "Existence of groundstates for a class of nonlinear Choquard equations in the plane," Advanced Nonlinear Studies, vol. 17, no. 3, pp. 581-594, 2017.

[19] Y. Sun, L. Liu, and Y. Wu, "The existence and uniqueness of positive monotone solutions for a class of nonlinear Schrödinger equations on infinite domains," Journal of Computational and Applied Mathematics, vol. 321, pp. 478-486, 2017.

[20] X. Du and A. Mao, "Existence and multiplicity of nontrivial solutions for a class of semilinear fractional Schrödinger equations," Journal of Function Spaces, vol. 7, 2017.

[21] A. Qian, "Infinitely many sign-changing solutions for a Schrödinger equation," Advances in Difference Equations, 2011:39, 6 pages, 2011.

[22] F. Gao and M. Yang, "On nonlocal Choquard equations with Hardy-Littlewood-Sobolev critical exponents," Journal of Mathematical Analysis and Applications, vol. 448, no. 2, pp. 10061041, 2017.

[23] F. Gao and M. Yang, "Brezis-Nirenberg type critical problem for nonlinear Choquard equation," Science China Mathematics.

[24] F. Gao and M. Yang, "A strongly indefinite Choquard equation with critical exponent due to the Hardy-Littlewood-Sobolev inequality," Communications in Contemporary Mathematics, vol. 20, no. 4, 1750037, 22 pages, 2018.

[25] Q. Li and Z. Yang, "Multiple positive solutions for a quasilinear elliptic system with critical exponent and sign-changing weight," Computers \& Mathematics with Applications. An International Journal, vol. 67, no. 10, pp. 1848-1863, 2014.

[26] X. He, A. Qian, and W. Zou, "Existence and concentration of positive solutions for quasi-linear Schrodinger equations with critical growth," Nonlinearity, vol. 26, no. 12, pp. 3137-3168, 2013.

[27] T. Li and T.-f. Wu, "Multiple positive solutions for a Dirichlet problem involving critical Sobolev exponent," Journal of Mathematical Analysis and Applications, vol. 369, no. 1, pp. 245-257, 2010.

[28] T. Mukherjee and K. Sreenadh, "Positive solutions for nonlinear Choquard equation with singular nonlinearity," Complex Variables and Elliptic Equations. An International Journal, vol. 62, no. 8, pp. 1044-1071, 2017.

[29] J. Liu and A. Qian, "Ground state solution for a SchrödingerPoisson equation with critical growth," Nonlinear Analysis: Real World Applications, vol. 40, pp. 428-443, 2018.

[30] J. Zhang, Z. Lou, Y. Ji, and W. Shao, "Ground state of Kirchhoff type fractional Schrödinger equations with critical growth," Journal of Mathematical Analysis and Applications, vol. 462, no. 1 , pp. 57-83, 2018.

[31] J. Seok, "Nonlinear Choquard equations involving a critical local term," Applied Mathematics Letters, vol. 63, pp. 77-87, 2017.

[32] Z. Shen, F. Gao, and M. Yang, "Ground states for nonlinear fractional Choquard equations with general nonlinearities," Mathematical Methods in the Applied Sciences, vol. 39, no. 14, pp. 4082-4098, 2016.
[33] C.-L. Xiang, "Uniqueness and nondegeneracy of ground states for Choquard equations in three dimensions," Calculus of Variations and Partial Differential Equations, vol. 55, no. 6, Art. 134, 25 pages, 2016.

[34] H. Ye, "Mass minimizers and concentration for nonlinear Choquard equations in RN," Topological Methods in Nonlinear Analysis, vol. 48, no. 2, pp. 393-417, 2016.

[35] H. Zhang, J. Xu, and F. Zhang, "Existence and multiplicity of solutions for a generalized Choquard equation," Computers \& Mathematics with Applications. An International Journal, vol. 73, no. 8, pp. 1803-1814, 2017.

[36] H. Zhang, J. Xu, and F. Zhang, "Bound and ground states for a concave-convex generalized Choquard equation," Acta Applicandae Mathematicae, vol. 147, pp. 81-93, 2017.

[37] G. A. Afrouzi and K. J. Brown, "Positive mountain pass solutions for a semilinear elliptic equation with a sign-changing weight function," Nonlinear Analysis. Theory, Methods \& Applications. An International Multidisciplinary Journal, vol. 64, no. 3, pp. 409-416, 2006.

[38] T.-S. Hsu and H.-L. Lin, "Multiple positive solutions for singular elliptic equations with concave-convex nonlinearities and sign-changing weights," Boundary Value Problems, Article ID 584203, pp. 1-17, 2009.

[39] T.-f. Wu, "Three positive solutions for Dirichlet problems involving critical Sobolev exponent and sign-changing weight," Journal of Differential Equations, vol. 249, no. 7, pp. 1549-1578, 2010.

[40] J. Ali and R. Shivaji, "Existence results for classes of Laplacian systems with sign-changing weight," Applied Mathematics Letters, vol. 20, no. 5, pp. 558-562, 2007.

[41] C.-y. Chen and T.-f. Wu, "Multiple positive solutions for indefinite semilinear elliptic problems involving a critical Sobolev exponent," Proceedings of the Royal Society of Edinburgh, Section: A Mathematics, vol. 144, no. 4, pp. 691-709, 2014.

[42] C.-y. Chen, Y.-c. Kuo, and T.-f. Wu, “The Nehari manifold for a Kirchhoff type problem involving sign-changing weight functions," Journal of Differential Equations, vol. 250, no. 4, pp. 1876-1908, 2011.

[43] G. Dai and X. Han, "Global bifurcation and nodal solutions for fourth-order problems with sign-changing weight," Applied Mathematics and Computation, vol. 219, no. 17, pp. 9399-9407, 2013.

[44] H. Fan, "Multiple positive solutions for semi-linear elliptic systems with sign-changing weight," Journal of Mathematical Analysis and Applications, vol. 409, no. 1, pp. 399-408, 2014.

[45] S. Goyal and K. Sreenadh, "Existence of multiple solutions of $\mathrm{p}$-fractional Laplace operator with sign-changing weight function," Advances in Nonlinear Analysis, vol. 4, no. 1, pp. 37$58,2015$. 


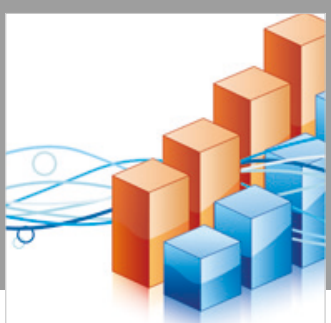

Advances in

Operations Research

\section{-n-m}
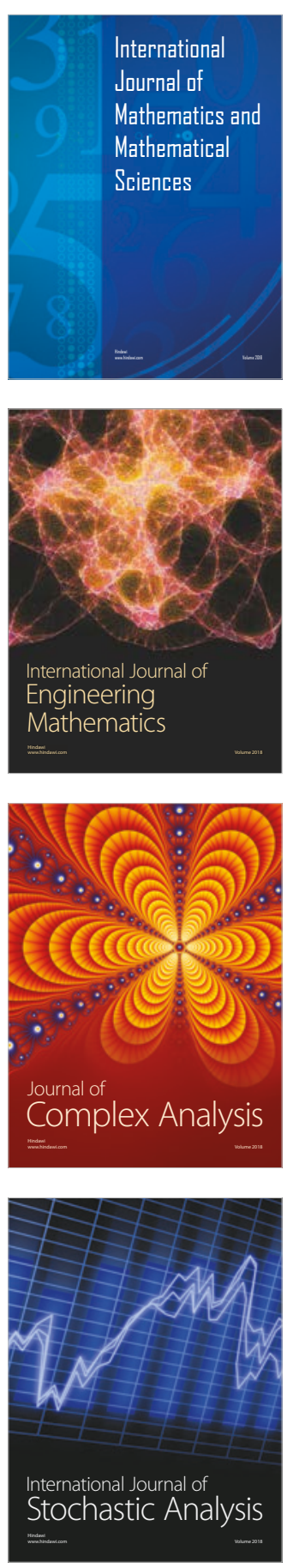
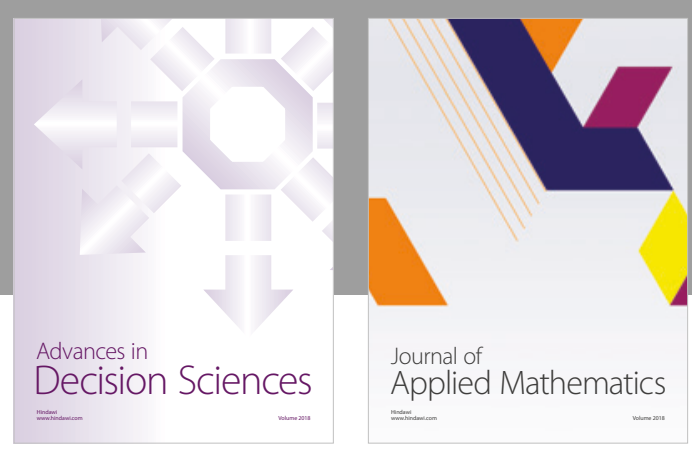

Journal of

Applied Mathematics
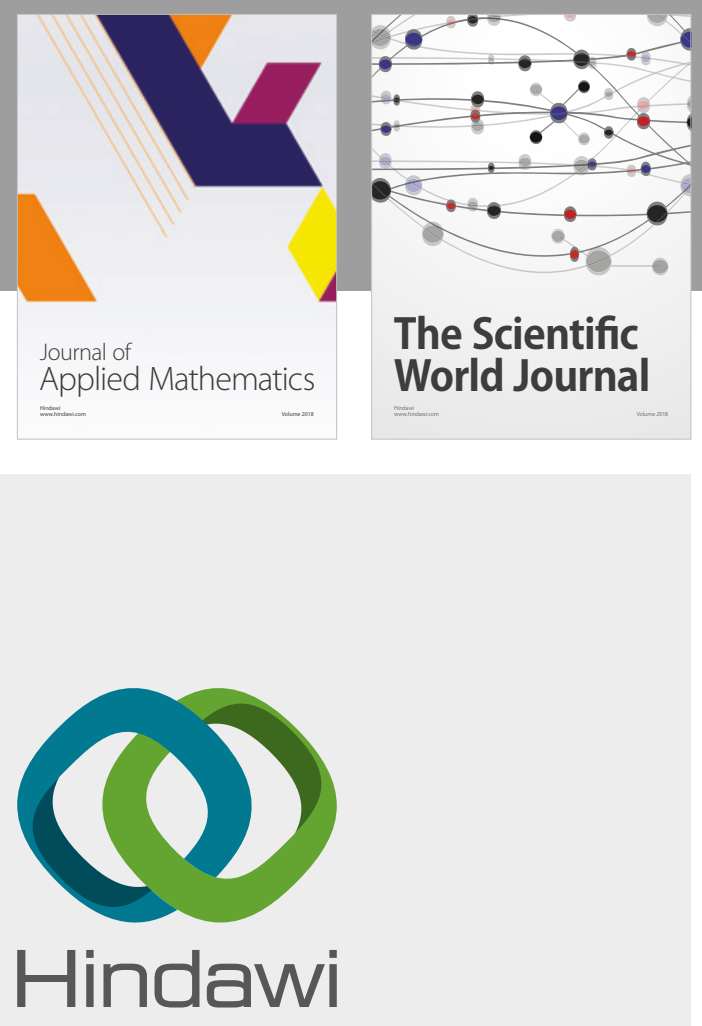

Submit your manuscripts at

www.hindawi.com

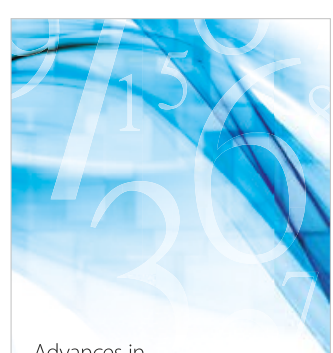

Advances in
Numerical Analysis
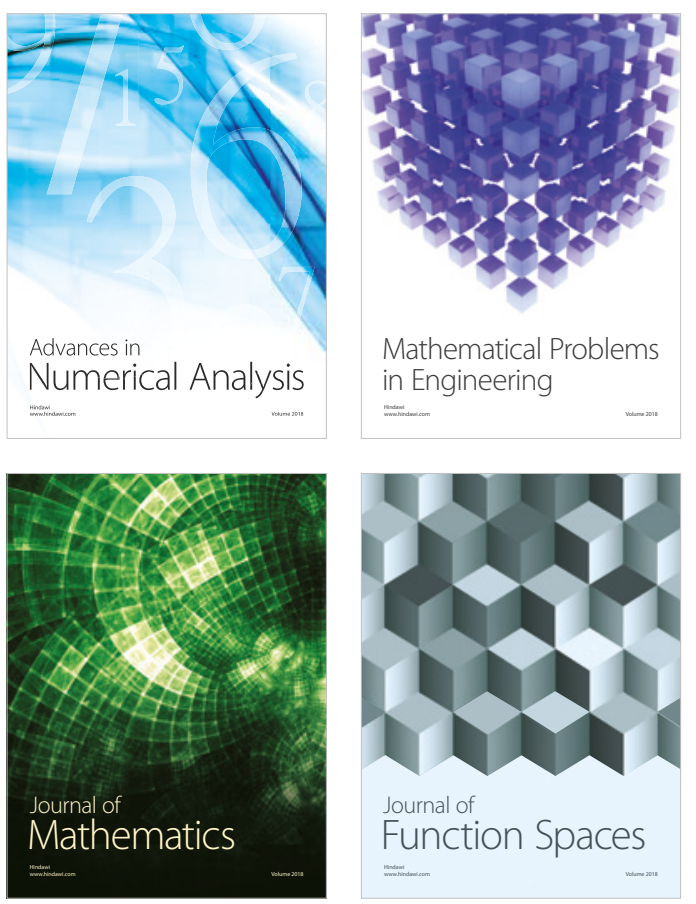

Mathematical Problems in Engineering

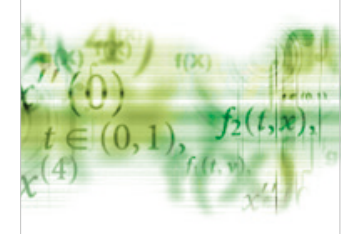

International Journal of

Differential Equations

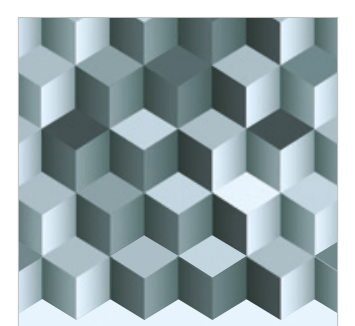

Journal of

Function Spaces

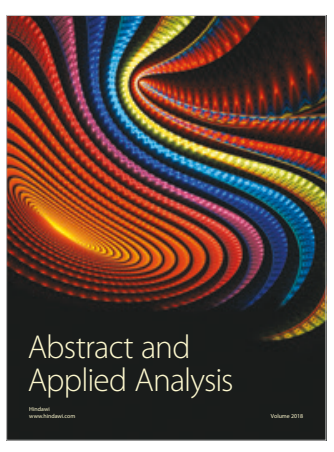

The Scientific

World Journal

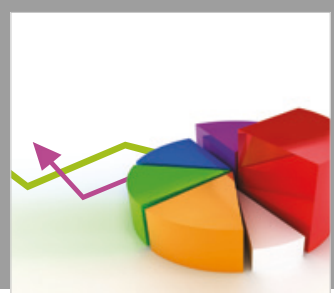

Journal of

Probability and Statistics
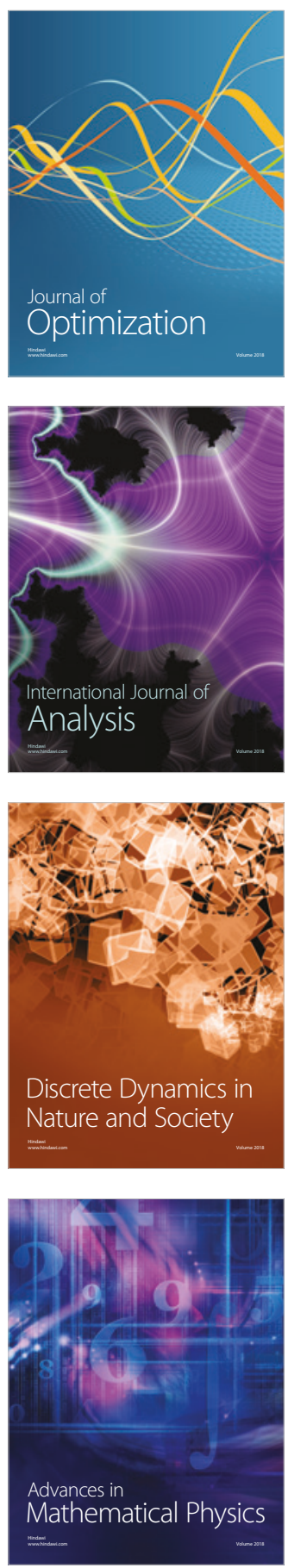